\title{
Joint waveform and guidance control optimisation for target rendezvous
}

\author{
Alessio Benavoli ${ }^{1}$, Alessio Balleri ${ }^{2}$ and Alfonso Farina ${ }^{3}$
}

\begin{abstract}
The algorithm developed in this paper jointly selects the optimal transmitted waveform and the control input so that a radar sensor on a moving platform with linear dynamics can reach a target by minimising a predefined cost. The cost proposed in this paper accounts for the energy of the transmitted radar signal, the energy of the platform control input and the relative position error between the platform and the target, which is a function of the waveform design and control input. Similarly to the Linear Quadratic Gaussian (LQG) control problem, we demonstrate that the optimal solution satisfies the separation principle between filtering and optimisation and, therefore, the optimum can be found analytically. The performance of the proposed solution is assessed with a set of simulations for a pulsed Doppler radar transmitting linearly frequency modulated chirps. Results show the effectiveness of the proposed approach for optimal waveform design and optimal guidance control.
\end{abstract}

Index Terms-Cognitive rendezvous, adaptive waveform design, cognitive radar, Fisher Information Matrix (FIM), CramérRao Lower Bound (CRLB), Linear Quadratic Gaussian control (LQG), Kalman Filter (KF).

\section{INTRODUCTION}

The improvement of technology and the pressing need for sensors to become increasingly more flexible, adaptive, and ultimately include some degree of cognition, is resulting in radar systems that are becoming more and more capable to adapt their parameters from scan to scan to optimise the sensor performance.

Cognitive radar systems are radar that continuously learn about the environment through active interactions with the environment itself and, as a result, continually update the receiver and the transmitting parameters, in an intelligent manner, with relevant information (the perception action cycle). The whole radar system is a dynamic closed feedback loop between the transmitter, environment and receiver. Cognition can be achieved with the use of prior knowledge on the environment, which represents the long-term memory of the receiver, and the short-term memory developed by the receiver on the fly.

In recent years, there has been growing research interest to develop solutions that can increase the level of cognition of radar systems [1] [2]. The largest portion of research published on cognitive radar presents solutions to adapt the transmitter parameters in response to the surrounding environment under

\footnotetext{
${ }^{1}$ A. Benavoli, Department of Computer Science and Information Systems (CSIS), University of Limerick, Ireland, e-mail: alessio.benavoli@ul.ie

${ }^{2}$ A. Balleri, Centre for Electronic Warfare, Information and Cyber, Cranfield University, Defence Academy of the UK, Shrivenham, SN6 8LA, e-mail: a.balleri@cranfield.ac.uk.

${ }^{3}$ A. Farina, LFIEEE, FIET, Fellow of EURASIP, Selex-ES (retired), Visiting Professor at UCL and Cranfield University, Rome, Italy, e-mail: alfonso.farina@outlook.it
}

the tracking framework. A technique to select the radiated waveform to improve target tracking performance was presented in [3]. The linear Kalman Filter (KF) is used in polar coordinates to track a target illuminated with a sensor system that measures the target range and radial velocity. The optimal waveform is selected by minimising the mean square tracking error and the volume of the tracking validation gate when the measurement covariance matrix is the Cramér-Rao Lower Bound (CRLB) of the joint estimation of range and velocity under the narrowband approximation. Adaptive waveform selection for a multistatic radar consisting of a transmitter and multiple receivers was investigated in [4]. The measurements of the relative bistatic target range, velocity and angle at each receiver are used to model the covariance matrices of the measurement noise in an Interacting Multiple Model - Extended Kalman Filter (IMM-EKF) and waveform selection is accomplished by minimising the tracking mean square error. Signal selection to improve tracking performance was studied in [5] to demonstrate the inappropriateness of selecting waveforms based on the accuracy of the measurements alone. Dynamic waveform selection based on the minimum expected value of the tracking Mean Square Error (MSE) was presented in [6] and applied to Generalised Frequency Modulated (GFM) chirp signals. A sequential Bayesian approach was proposed in [7] to automatically adapt the waveform design with an antenna array receiving multiple polarisations and in the presence of non-Gaussian clutter. A suboptimal, but less computationally expensive, algorithm was also proposed to select waveform parameters. This work was further generalised in [8] by separating the general principles of cognition from the specific applications to provide a flexible framework applicable to tracking problems. This was followed by a solution to design cost functions for fully adaptive radar in [9]. A method based on mutual information performance to select the waveform design for target tracking was proposed in [10]. This method accounts for realistic physical and statistical effects, including the earth's curvature and compound-Gaussian clutter, and was applied to co-located Multiple-Input-Multiple-Output (MIMO) radar transmitting wideband Orthogonal Frequency Division Multiplexing (OFDM) waveforms. Cognitive target tracking for Frequency Diverse Arrays (FDA) was presented in [11]. A solution to design a multibeam radar capable of adapting the transmitting peak power of each beam was presented in [12]. The transmit power of each beam is adapted by using a performance metric based on the Bayesian CRLB with Measurement Origin Uncertainty (MOU) which relies on prior knowledge predicted with the tracking recursive cycle. The algorithm works under the assumption that each beam 
tracks only a target at a time and includes the task of target detection and false alarms. Cognition was applied to a particle filter for tracking a manoeuvring target in [13]. The algorithm selects dynamically the number of particle filters and the transmitted waveform so to improve tracking accuracy and tracking efficiency under the assumption of a linear Gaussian system. Additional interesting references on these topics are [14] [15]. Recent work on cognitive radar has also investigated the problem of coexisting Orthogonal Frequency Division Multiplexing (OFDM) radar and OFDM communication base stations and proposed a solution to optimise the reduction of radar transmitted power so to maintain a predefined Mutual Information (MI) constraint [16].

Despite previous work on tracking by cognitive radar, very little research has been focused on how radar cognition can be applied to guidance control for target rendezvous. There has been prior work on optimal path planning investigating the optimal trajectories of multiple moving receivers, forming a multistatic radar network with a single adaptive transmitter, and tasked to track a moving target. The waveform and trajectories were selected so to minimise the trace of the covariance matrix of the KF [17]. However, in this work there is no attempt to achieve guidance control and there are no constraints on each receiving platform trajectory.

The key challenge of studying cognition applied to rendezvous problems, as it is proposed in this paper, is the requirement of optimal solutions that handle estimation theory and platform guidance control jointly to ensure the trajectory of the platform ends at the target. The ultimate goal is the development of a radar platform which can emulate the behaviour of an echolocating bat that diversifies the echolocation call parameters along its trajectory to successfully catch an insect [18] [19] [20].

Optimal Waveform Diversity (WD) and platform control for target rendezvous are techniques which have been mostly researched separately in the past. The concept of duality between estimation and control was first investigated by Kalman in [21]. One of the first papers on optimal guidance for rendezvous investigated the case of a sensor on the ground that delivered optimal guidance to the interceptor on the basis of the estimated trajectories of the interceptor and the target to reach [22]. The sensor transmitted a suitable waveform which, however, did not change during the task.

An algorithm to automatically select the transmitted waveform design from scan to scan to guide an airborne platform towards a target was presented in [23]. The fore-active control technique proposed in [3] was used to select the transmitted waveform, so to minimise the innovation matrix (or residual) of the KF, and a standard Linear Quadratic Gaussian control (LQG) technique was employed to guide the platform towards the target. However, in this work, the fore-active technique and LQG control were applied independently with no attempt to demonstrate the existence and find the joint optimal solution.

The contribution of this paper is twofold. Firstly, the analytical demonstrations presented in this paper represent an important contribution to the linear KF theory with a generalisation of the LQG control approach. Secondly, the paper presents a new optimal solution to achieve joint optimal WD and guidance control for rendezvous. The algorithm minimises a cost function that depends on both the parameters of the transmitted radar waveform and the input guidance control so that the waveform and guidance control input selection, under suitable assumptions, can be truly, jointly and simultaneously optimised. We demonstrate for the first time that for a linear Gaussian system, when the measurement noise is independent of the state, the joint optimisation problem can be simplified into two independent optimisations, one that depends only on the waveform parameters and the other that depends solely on the control input. The solution is found in a closed-form and therefore the proposed algorithm has comparable computational complexity to existing cognitive solutions proposed for tracking. The mathematical demonstrations in this paper also rigorously prove that the algorithm presented in [23] is suboptimal and provide a modified optimal solution.

\section{LIST OF MAIN SYMBOLS}

\begin{tabular}{|c|c|c|}
\hline $\mathbf{e}_{k}$ & $N_{\mathrm{X}} \times 1$ & State vector of relative dynamics \\
\hline$\hat{\mathbf{e}}_{k}$ & $N_{\mathrm{X}} \times 1$ & Mean value of Kalman Filter \\
\hline $\mathbf{w}_{k}$ & $N_{w} \times 1$ & State forcing noise \\
\hline $\mathbf{u}_{k}$ & $N_{u} \times 1$ & Control input \\
\hline $\mathbf{z}_{k}$ & $N_{\mathrm{Z}} \times 1$ & Measurement vector \\
\hline$\nu_{k}$ & $N_{\mathrm{Z}} \times 1$ & Additive noise for the measurement \\
\hline $\mathbf{P}_{k \mid k}$ & $N_{\mathrm{X}} \mathrm{x} N_{\mathrm{x}}$ & State error covariance \\
\hline $\mathbf{P}_{k \mid k-1}$ & $N_{\mathrm{X}} \mathrm{x} N_{\mathrm{x}}$ & Prediction of state error covariance \\
\hline $\mathbf{Q}_{k}$ & $N_{\mathrm{X}} \mathrm{x} N_{\mathrm{x}}$ & Covariance of state forcing noise \\
\hline $\mathbf{N}_{k}$ & $N_{\mathrm{Z}} \times N_{\mathrm{Z}}$ & Covariance of measurement noise \\
\hline $\mathbf{F}_{k}$ & $N_{\mathrm{X}} \mathrm{x} N_{\mathrm{X}}$ & State transition matrix \\
\hline $\mathbf{M}_{k}$ & $N_{\mathrm{x}} \mathrm{x} N_{\mathrm{x}}$ & Cost function matrix \\
\hline $\mathbf{U}_{k}$ & $N_{\mathrm{X}} \mathrm{x} N_{\mathrm{x}}$ & Dynamic Riccati equation matrix \\
\hline $\mathbf{S}_{k}$ & $N_{\mathrm{Z}} \times N_{\mathrm{z}}$ & Residual covariance matrix \\
\hline $\mathbf{H}_{k}$ & $N_{\mathrm{Z}} \times N_{\mathrm{x}}$ & Measurement matrix \\
\hline $\mathbf{K}_{k}$ & $N_{\mathrm{X}} \times N_{\mathrm{Z}}$ & Kalman gain \\
\hline $\mathbf{E}_{k}$ & $N_{\mathrm{X}} \mathrm{x} N_{\mathrm{X}}$ & Weight matrix of the cost function \\
\hline $\mathbf{R}_{k}$ & $N_{\mathrm{u}} \times N_{\mathrm{u}}$ & Weight matrix of the cost function \\
\hline $\mathbf{L}_{k}$ & $N_{\mathrm{u}} \times N_{\mathrm{x}}$ & Feedback gain matrix \\
\hline $\mathbf{B}_{k}$ & $N_{\mathrm{X}} \mathrm{x} N_{\mathrm{u}}$ & Control transition matrix \\
\hline $\boldsymbol{\theta}_{k}$ & $N_{\theta} \times 1$ & Vector of waveform parameters \\
\hline $\mathbf{I}_{N \times N}$ & $N \times N$ & Identity matrix \\
\hline
\end{tabular}

\section{THEORETICAL FRAMEWORK}

The goal of optimal control theory is to operate/regulate a dynamic system at minimum cost. The case where the system dynamics is linear and the cost is a quadratic function is called the linear quadratic (LQ) control problem. When the linear systems are driven by additive white Gaussian noise, the problem is known as an LQG control problem. The goal of this section is to extend the LQG control problem to the case where, at each time instant, the parameters of the measurement sensor can be regulated too. These parameters may determine the accuracy, but also the energy consumption of the sensor. Hence, we aim to jointly select the control inputs and sensing parameters optimally, by minimizing the LQG quadratic cost function together with an additive sensing cost term that penalises the sensor. 
Consider a general linear system dynamics

$$
\mathbf{e}_{k}=\mathbf{F}_{k-1} \mathbf{e}_{k-1}+\mathbf{B}_{k-1} \mathbf{u}_{k-1}+\mathbf{w}_{k-1}
$$

where $\mathbf{e}_{k}$ is the state vector, $\mathbf{F}_{k-1}$ is the matrix dynamics and $\mathbf{w}_{k-1}$ is a zero-mean Gaussian random perturbation with covariance matrix $\mathbf{Q}_{k-1}$. The term $\mathbf{B}_{k-1} \mathbf{u}_{k-1}$ is the control action at each step: the input vector control $\mathbf{u}_{k-1}$ is combined linearly with a matrix $\mathbf{B}_{k-1}$ before being applied to the dynamics equations. At each time $k$ a sensor senses the environment. The accuracy of the sensor is defined by a vector of parameters $\boldsymbol{\theta}_{k}$ (e.g., for radar sensors, the parameters are duration, bandwidth etc.), to obtain a linear measurement

$$
\mathbf{z}_{k}=\mathbf{H}_{k} \mathbf{e}_{k}+\boldsymbol{\nu}_{k}\left(\boldsymbol{\theta}_{k}\right)
$$

of the state $\mathbf{e}_{k}$, as in [3]. The matrix that maps the state into the measurement is $\mathbf{H}_{k}$ and $\boldsymbol{\nu}_{k}\left(\boldsymbol{\theta}_{k}\right)$ is a zero-mean Gaussian random process with covariance matrix $\mathbf{N}\left(\boldsymbol{\theta}_{k}\right)$ which depends on the parameters $\boldsymbol{\theta}_{k}$. The goal of the platform is to minimise a quadratic cost function

$$
J\left(\mathbf{e}_{0}, \mathbf{u}_{0: N-1}, \boldsymbol{\theta}_{1: N}\right)=\mathscr{E}\left(\sum_{k=0}^{N} \ell\left(k, \mathbf{e}_{k}, \mathbf{u}_{k}, \boldsymbol{\theta}_{k}\right)\right)
$$

with respect to $\mathbf{u}_{0: N-1}=\left\{\mathbf{u}_{0}, \ldots, \mathbf{u}_{N-1}\right\}$ and $\boldsymbol{\theta}_{1: N}=$ $\left\{\boldsymbol{\theta}_{1}, \ldots, \boldsymbol{\theta}_{N}\right\}$, over the horizon $N$, with

$\left\{\ell\left(k, \mathbf{e}_{k}, \mathbf{u}_{k}, \boldsymbol{\theta}_{\boldsymbol{k}}\right)=\mathbf{e}_{k}^{T} \mathbf{E}_{k} \mathbf{e}_{k}+\mathbf{u}_{k}^{T} \mathbf{R}_{k} \mathbf{u}_{k}+\alpha_{k} \Psi_{s}\left(\boldsymbol{\theta}_{\boldsymbol{k}}\right) \quad k<N\right.$ $\left\{\ell\left(N, \mathbf{e}_{N}, \mathbf{u}_{N}, \boldsymbol{\theta}_{N}\right)=\mathbf{e}_{N}^{T} \mathbf{E}_{N} \mathbf{e}_{N}+\operatorname{Tr}\left(\mathbf{E}_{N} \mathbf{P}_{N \mid N}\right)+\alpha_{N} \Psi_{s}\left(\boldsymbol{\theta}_{N}\right)\right.$

where

- $\mathscr{E}(\ldots)$ denotes the expectation w.r.t. the initial state and the sequence of measurement and process noises;

- $\mathbf{E}_{k}, \mathbf{R}_{k}$ are suitable Positive Semi-Definite (PSD) matrices that define the weights of the cost function: the control effort is weighted by the matrices $\mathbf{R}_{k}$, while the control effectiveness by $\mathbf{E}_{k}$;

- $\alpha_{k} \geq 0$ are additional scalar weights of the cost function that penalise an additional sensing cost term $\Psi_{s}\left(\theta_{k}\right) \geq 0$;

- the meaning of term $\operatorname{Tr}\left(\mathbf{E}_{N} \mathbf{P}_{N \mid N}\right)$ will be clarified in the next section. For the moment, we can just consider it as a further additive term in the cost function;

The control goal is therefore to keep $\mathbf{e}_{k}$ close to $\mathbf{0}$, especially, at the final time $N$, using little control effort $\mathbf{u}_{k}$ and little sensing cost $\Psi_{s}\left(\boldsymbol{\theta}_{\boldsymbol{k}}\right)$.

Therefore, the dependence of the cost function on $\boldsymbol{\theta}_{1: N}$ is twofold: (i) the sensing cost term $\Psi_{s}\left(\boldsymbol{\theta}_{\boldsymbol{k}}\right)$; (ii) the measurement noise $\left(\boldsymbol{\nu}_{k}\left(\boldsymbol{\theta}_{k}\right)\right)$ that contributes to the covariance matrix of the joint multivariate Gaussian distribution w.r.t. which the expectation in (3) is taken. For fixed sensor parameters (fixed $\boldsymbol{\theta}_{1: N}$ ) and $\alpha_{k}=0$, the optimal control problem in (3) coincides with the LQG regulator problem, whose optimal solution is well-known, see for instance [24, Ch.7]. Note in fact that, in this setting, the cost function is quadratic in the state and control inputs. In this paper, by introducing the additional optimisation variables $\boldsymbol{\theta}_{1: N}$, we give the possibility to the interceptor to both control its trajectory (through $\mathbf{u}_{0: N-1}$ ) and the sensor sensing parameters generation (through $\boldsymbol{\theta}_{1: N}$ ).

\section{TheOREM OF JOINT WAVEFORM AND GUIDANCE CONTROL OPTIMISATION (JWGCO)}

Consider the linear Gaussian system (1) and the quadratic performance cost (3)-(4). Our goal in this section is to find a joint sensing parameters selection and guidance control sequence $\left(\mathbf{u}_{0: N-1}, \boldsymbol{\theta}_{1: N}\right)$ that minimizes the performance cost (3). For fixed sensor parameters $\boldsymbol{\theta}_{1: N}$, the optimal sequence $\mathbf{u}_{0: N-1}$ can be found by solving the LQG control problem. ${ }^{1}$ In this case, an elegant solution can be obtained in closedform (see for instance [24, Ch.7]). In the following Theorem 1 , we extend this known result to the case in which the sensor parameters change and can be optimised with time.

Theorem 1. Under the assumption that the noises $\boldsymbol{\nu}_{k}\left(\boldsymbol{\theta}_{k}\right)$, $\mathbf{w}_{k}$ and the initial state $\mathbf{e}_{0}$ are jointly independent for $k=$ $0,1,2, \ldots$, the optimal solution of the problem (3)-(4) assuming the dynamics (1) and the measurement equation (2) is $\mathbf{u}_{i}^{*}=-\mathbf{L}_{i} \hat{\mathbf{e}}_{i}$ and $\boldsymbol{\theta}_{i}^{*}=\arg \min _{\boldsymbol{\theta}_{i}} \operatorname{Tr}\left(\mathbf{M}_{i} \mathbf{P}_{i \mid i}\right)+\alpha_{i} \Psi_{s}\left(\boldsymbol{\theta}_{i}\right)$ with

$$
\mathbf{M}_{i}= \begin{cases}\mathbf{S}_{N}, & \text { for } i=N \\ \left(\mathbf{E}_{i}+\mathbf{F}_{i}^{T} \mathbf{S}_{i+1} \mathbf{F}_{i}-\mathbf{S}_{i}\right), & \text { for } i=1, \ldots, N-1\end{cases}
$$

and

$$
\hat{\mathbf{e}}_{i+1}=\mathbf{F}_{i} \hat{\mathbf{e}}_{i}+\mathbf{B}_{i} \mathbf{u}_{i}+\mathbf{K}_{i+1}\left(\mathbf{z}_{i+1}-\mathbf{H}_{i+1}\left(\mathbf{F}_{i} \hat{e}_{i}+\mathbf{B}_{i} \mathbf{u}_{i}\right)\right)
$$

with $\hat{\mathbf{e}}_{0}=\mathscr{E}\left[\mathbf{e}_{0}\right]$. The Kalman gain equals

$$
\mathbf{K}_{i}=\mathbf{P}_{i \mid i-1} \mathbf{H}_{i}^{\mathrm{T}}\left(\mathbf{H}_{i} \mathbf{P}_{i \mid i-1} \mathbf{H}_{i}^{\mathrm{T}}+\mathbf{N}_{i}\left(\boldsymbol{\theta}_{i}\right)\right)^{-1}
$$

where $\mathbf{P}_{i \mid i}$ is determined by the following Riccati matrix difference equation that runs forward in time:

$$
\mathbf{P}_{i \mid i}=\mathbf{P}_{i \mid i-1}-\mathbf{K}_{i} \mathbf{H}_{i} \mathbf{P}_{i \mid i-1}
$$

where

$$
\mathbf{P}_{i \mid i-1}=\mathbf{F}_{i-1} \mathbf{P}_{i-1 \mid i-1} \mathbf{F}_{i-1}^{\mathrm{T}}+\mathbf{Q}_{i-1}
$$

and $\mathbf{P}_{0 \mid 0}=\mathscr{E}\left(\left(\mathbf{e}_{0}-\hat{\mathbf{e}}_{0}\right)\left(\mathbf{e}_{0}-\hat{\mathbf{e}}_{0}\right)^{\mathrm{T}}\right)$. The feedback gain matrix equals

$$
\mathbf{L}_{i}=\left(\mathbf{B}_{i}^{\mathrm{T}} \mathbf{S}_{i+1} \mathbf{B}_{i}+\mathbf{R}_{i}\right)^{-1} \mathbf{B}_{i}^{\mathrm{T}} \mathbf{S}_{i+1} \mathbf{F}_{i}
$$

where $\mathbf{S}_{i}$ is determined by the following Riccati matrix difference equation that runs backward in time:

$$
\mathbf{S}_{i}=\mathbf{F}_{i}^{\mathrm{T}}\left(\mathbf{S}_{i+1}-\mathbf{S}_{i+1} \mathbf{B}_{i}\left(\mathbf{B}_{i}^{\mathrm{T}} \mathbf{S}_{i+1} \mathbf{B}_{i}+\mathbf{R}_{i}\right)^{-1} \mathbf{B}_{i}^{\mathrm{T}} \mathbf{S}_{i+1}\right) \mathbf{F}_{i}+\mathbf{E}_{i}
$$

with $\mathbf{S}_{N}=\mathbf{E}_{N}$. Finally the optimal cost is

$$
\begin{aligned}
& \min _{\mathbf{u}_{0: N-1}, \boldsymbol{\theta}_{1: N}} J\left(\mathbf{e}_{0}, \mathbf{u}_{0: N-1}, \boldsymbol{\theta}_{1: N}\right)=\mathscr{E}\left(\mathbf{e}_{0}^{T} \mathbf{S}_{0} \mathbf{e}_{0}\right)+\operatorname{Tr}\left(\mathbf{M}_{N} \mathbf{P}_{N \mid N}^{*}\right) \\
& +\alpha_{N} \Psi_{s}\left(\boldsymbol{\theta}_{N}^{*}\right)+\sum_{i=0}^{N-1} \operatorname{Tr}\left(\mathbf{S}_{i+1} \mathbf{Q}_{i}\right)+\operatorname{Tr}\left(\mathbf{M}_{i} \mathbf{P}_{i \mid i}^{*}\right)+\alpha_{i} \Psi_{s}\left(\boldsymbol{\theta}_{i}^{*}\right)
\end{aligned}
$$

where $\mathbf{P}_{i \mid i}^{*}$ is the value of the covariance matrix $\mathbf{P}_{i \mid i}$ when computed with the optimal measurement noise covariance $\mathbf{N}_{i}\left(\boldsymbol{\theta}_{i}^{*}\right)$.

A detailed proof of Theorem 1 can be found in Appendix A, Appendix B and Appendix C.

\footnotetext{
${ }^{1}$ The difference w.r.t. the LQG problem is the term $\operatorname{Tr}\left(\mathbf{E}_{N} \mathbf{P}_{N \mid N}\right)$, that does not depend on $\mathbf{u}_{1: N}$ and, therefore, does not change the LQG solution. By removing this last term and forcing $\alpha_{k}=0$ for or $k \leq N$ and $\mathbf{E}_{k}=0$ for $k<N$, the cost function corresponds to the LQG cost exploited in [23].
} 
It can be noticed that the optimal guidance control law resembles the classic LQG regulation: the only difference is that the covariance matrix of the KF depends on the optimal sensor parameters. This is illustrated in the block diagram in Figure 1. One of the remarkable properties of LQG regulation is the separation principle: the filtering stage is not affected by the regulation objective and conversely. This property is kept by the current solution and this allows us to perform the two optimizations (w.r.t. $\boldsymbol{\theta}_{1: N}$ and $\mathbf{u}_{0: N-1}$ ) independently. However, this does not mean that the optimal values (resulting from the optimisations) are independent. They both depend, in a non-trivial way, on the mean and covariance of the KF and on the matrices of the cost function. Moreover, the optimal action and optimal sensing at time $i$ change the trajectory of the interceptor and the sensing accuracy at time $i+1$ leading to a closed-loop system. This allows us to jointly trade-off between the control effort, the control effectiveness and the sensing cost in an optimal way.

In the LQG regulator an interesting topic is the analysis of the limiting behaviour as the regulation $N$ horizon goes to infinity. For fixed sensor parameters (fixed $\boldsymbol{\theta}_{1}=\boldsymbol{\theta}_{2}=\cdots=\boldsymbol{\theta}_{N}$ ), no energy term $\left(\alpha_{k}=0\right)$ and a time-invariant system (i.e., $\mathbf{F}, \mathbf{B}, \mathbf{Q}, \mathbf{H}, \mathbf{N}$ are time-invariant, also $\mathbf{N}=\mathbf{N}\left(\boldsymbol{\theta}_{i}\right)$ being constant), we recall the following result from LQG regulation [24].

Proposition 1. Consider a time-invariant linear Gaussian plant (1),(2) and the quadratic performance index (3)-(4) with time-invariant weight matrices, fixed $\boldsymbol{\theta}_{1}=\boldsymbol{\theta}_{2}=\cdots=\boldsymbol{\theta}_{N}$ and no energy terms $\left(\alpha_{k}=0\right)$. Let $(\mathbf{F}, \mathbf{B})$ and $(\mathbf{F}, \mathbf{G})$ (with $\left.\mathbf{G}=\mathbf{Q}^{\mathbf{1} / \mathbf{2}}\right)$ be stabilisable and $(\mathbf{F}, \mathbf{H})$ be detectable. Then, as $N \rightarrow \infty$, the $L Q G$ optimal regulation law equals $\mathbf{u}_{i}^{*}=-\mathbf{L} \hat{\mathbf{e}}_{i}$ where $\mathbf{L}$ is the constant-feedback matrix

$$
\mathbf{L}=\left(\mathbf{B}^{\mathrm{T}} \mathbf{S B}+\mathbf{R}\right)^{-1} \mathbf{B}^{\mathrm{T}} \mathbf{S F}
$$

where $\mathbf{S}$ is the solution of the ARE (Algebraic Riccati Equation):

$$
\mathbf{S}=\mathbf{F}^{\mathrm{T}}\left(\mathbf{S}-\mathbf{S B}\left(\mathbf{B}^{\mathrm{T}} \mathbf{S B}+\mathbf{R}\right)^{-1} \mathbf{B}^{\mathrm{T}} \mathbf{S}\right) \mathbf{F}+\mathbf{E}
$$

Further, $\hat{\mathbf{e}}_{i}$ is generated by the steady-state KF with Kalman gain:

$$
\mathbf{K}=\mathbf{P} \mathbf{H}^{\mathrm{T}}\left(\mathbf{H P H}^{\mathrm{T}}+\mathbf{N}\right)^{-1}
$$

where $\mathbf{P}$ is the solution of the ARE:

$$
\mathbf{P}=\mathbf{F}\left(\mathbf{P}-\mathbf{P H}^{\mathrm{T}}\left(\mathbf{H} \mathbf{P} \mathbf{H}^{\mathrm{T}}+\mathbf{N}\right)^{-1} \mathbf{H P}\right) \mathbf{F}^{\mathrm{T}}+\mathbf{Q}
$$

In our case, even for time-invariant systems, the matrix $\mathbf{N}\left(\boldsymbol{\theta}_{i}\right)$ is time-variant because of the dependence on the decision variables $\boldsymbol{\theta}_{i}$. Therefore, the previous Proposition does not hold. However, we can prove the following result.

Theorem 2. Consider the same assumptions as in Proposition 1 apart from that of fixed sensor parameters and assume that $\alpha_{1}=\cdots=\alpha_{n}=\alpha$. Then, as $N \rightarrow \infty$, the LQG optimal regulation law equals $\mathbf{u}_{i}^{*}=-\mathbf{L} \hat{\mathbf{e}}_{i}$ where $\mathbf{L}$ is the constantfeedback matrix given by (12)-(13). Moreover, let $\mathbf{M}$ be the weight matrix in (5) computed for $\mathbf{S}_{i}=\mathbf{S}$ and let us assume that there exists a solution $\mathbf{P}^{*}$ of the ARE (8) computed assuming a constant $\mathbf{N}\left(\boldsymbol{\theta}_{i}\right)=\mathbf{N}\left(\boldsymbol{\theta}^{*}\right)$ for $i=1,2, \ldots$ and such that

$$
\arg \min _{\boldsymbol{\theta}} \operatorname{Tr}(\mathbf{M} \tilde{\mathbf{P}})+\alpha \Psi_{s}(\boldsymbol{\theta})=\boldsymbol{\theta}^{*}
$$

with $\tilde{\mathbf{P}}=\mathbf{P}^{*}-\mathbf{P}^{*} \mathbf{H}_{i}^{\mathrm{T}}\left(\mathbf{H} \mathbf{P}^{*} \mathbf{H}^{\mathrm{T}}+\mathbf{N}(\boldsymbol{\theta})\right)^{-1} \mathbf{H} \mathbf{P}^{*}$. Then (3)-(4) admits a steady-state solution that coincides with that of the $L Q G$ regulator computed for $\mathbf{N}=\mathbf{N}\left(\boldsymbol{\theta}^{*}\right)$.

A detailed demonstration is given in Appendix D.

Theorem 2 proves that a steady solution may exist also for the optimal joint sensing parameters selection and guidance control problem. However, it is not guaranteed that the steady solution is unique, since there may be different values of $\theta^{*}$ that satisfy (16).

Finally, it is worth noting that in this work, we are assuming the sensor parameters can vary continuously (in intervals) but, in practice, they may be constrained to only assuming discrete values. In this case, the optimisation problem can be formulated as a mixed integer-nonlinear programming, the integer part being the optimisation of the waveform parameters $\arg \min _{\boldsymbol{\theta}_{i}} \operatorname{Tr}\left(\mathbf{M}_{i} \mathbf{P}_{i \mid i}\right)+\alpha_{i} \Psi_{s}\left(\boldsymbol{\theta}_{i}\right)$, that can be solved using either a brute-force search or a branch-bound algorithm [25] (depending on the number of points in the search space).

\section{MEASUREMENT NOISE COVARIANCE MATRIX}

We consider the case of a radar system that measures the relative range, radial velocity and angle with respect to the target in a polar coordinate system. The covariance matrix of the measurement noise depends on the elements of the vector $\boldsymbol{\theta}_{k}$ that describes the parameters of the transmitted waveform, such as duration, bandwidth and time-frequency curvature. The mathematical expression of the elements of $\boldsymbol{\theta}_{k}$ and the vector length depend on the waveform design taken under consideration. It is well known in the literature that, when the sensor measures range and radial velocity, the covariance matrix $\hat{\mathbf{N}}\left(\boldsymbol{\theta}_{k}\right)$ corresponds to the CRLB of the estimators of range and radial velocity [26] [27] [28]. The Fisher Information Matrix (FIM) for range $r$ and radial velocity $\dot{r}$ in the presence of noise with mean power $N_{0}$ can be expressed as

$$
\mathbf{J}^{(r, \dot{r})}=\mathbf{J}^{(\tau, v)} \circ\left(\begin{array}{cc}
\frac{4}{c^{2}} & \frac{4}{c \lambda} \\
\frac{4}{c \lambda} & \frac{4}{\lambda^{2}}
\end{array}\right)
$$

with

$$
\mathbf{J}^{(\tau, v)}=-\left.\operatorname{SNR}\left(\begin{array}{ll}
\frac{1}{2} \frac{\partial^{2}\left|\chi\left(\tau, v ; \boldsymbol{\theta}_{k}\right)\right|^{2}}{\partial \tau^{2}} & \frac{1}{2} \frac{\partial^{2}\left|\chi\left(\tau, v ; \boldsymbol{\theta}_{k}\right)\right|^{2}}{\partial \tau \partial v} \\
\frac{1}{2} \frac{\partial^{2}\left|\chi\left(\tau, v ; \boldsymbol{\theta}_{k}\right)\right|^{2}}{\partial v \partial \tau} & \frac{1}{2} \frac{\partial^{2}\left|\chi\left(\tau, v ; \boldsymbol{\theta}_{k}\right)\right|^{2}}{\partial v^{2}}
\end{array}\right)\right|_{\tau, v=0}
$$

where $\circ$ is the Hadamard product, $c$ is the speed of propagation, $\lambda$ is the wavelength, $\mathrm{SNR}=2 E_{R} / N_{0}$ is the Signal to Noise Ratio at the receiver and $\chi_{k}(\tau, v)$ is the normalised narrowband Complex Ambiguity Function (CAF) of the signal $\sqrt{E_{R}} s\left(t ; \boldsymbol{\theta}_{k}\right)$ of energy $E_{R}$ defined as

$$
\chi\left(\tau, v ; \boldsymbol{\theta}_{k}\right)=\int_{-\infty}^{\infty} s\left(t ; \boldsymbol{\theta}_{k}\right) s^{*}\left(t+\tau ; \boldsymbol{\theta}_{k}\right) e^{j 2 \pi v t} d t
$$

where $\tau$ and $v$ represent the time delay and the Doppler, respectively. The covariance matrix of the measurement noise, 


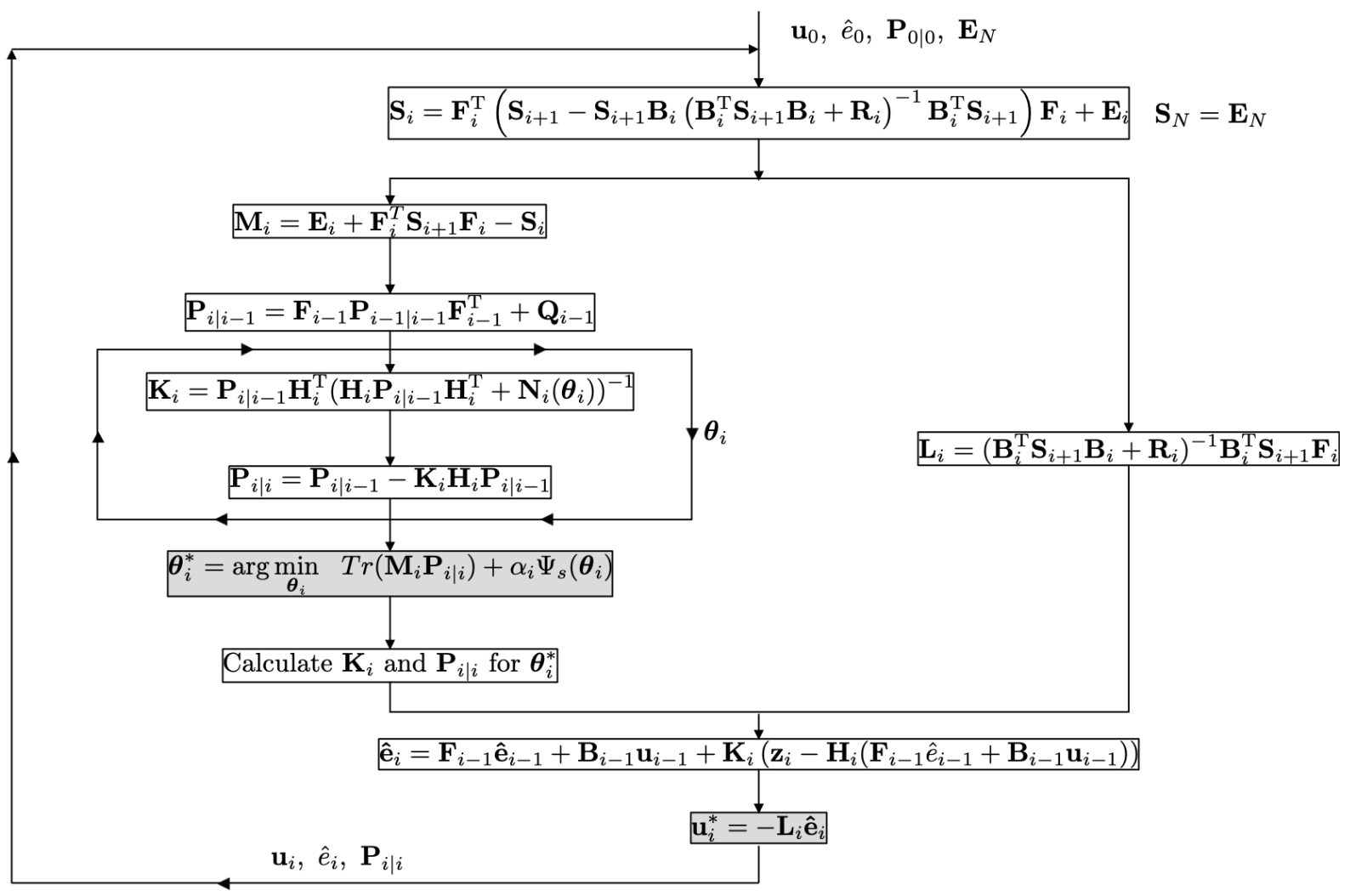

Fig. 1: Block diagram of the proposed JWGCO algorithm. The two highlighted boxes represent the two optimised variables.

that is the CRLB, is therefore obtained by taking the inverse of the FIM

$$
\hat{\mathbf{N}}\left(\boldsymbol{\theta}_{k}\right)=\left[\mathbf{J}^{(r, \dot{r})}\right]^{-1}
$$

The radar measurement of target angle at narrowband is assumed to be independent of the measurements of range and velocity and therefore the final measurement noise covariance matrix is

$$
\mathbf{N}\left(\boldsymbol{\theta}_{k}\right)=\left(\begin{array}{cc}
\hat{\mathbf{N}}\left(\boldsymbol{\theta}_{k}\right) & 0 \\
\mathbf{0}_{2 \times 2} & \sigma_{\theta}^{2}
\end{array}\right)
$$

where $\sigma_{\theta}^{2}$ is the variance of the measurement of target angle which, in general, is a function of SNR, antenna design (i.e. beamshape) and beampointing. In this paper, we study the case of a sensor that transmits a train of $N_{P}$ chirps with a Gaussian envelope and with duration $T=2 \lambda_{G}$ and bandwidth $B=2 b_{G} \lambda_{G} / \pi$ of the form

$$
s_{T}\left(t ; \boldsymbol{\theta}_{k}\right)=\sqrt{E_{s}} \frac{1}{\sqrt{N_{P}}} \sum_{i=0}^{N_{p}-1} x(t-i P R I)
$$

with

$$
x\left(t ; \boldsymbol{\theta}_{k}\right)=\left(\frac{1}{\pi \lambda_{G}^{2}}\right)^{1 / 4} e^{-\frac{t^{2}}{2 \lambda_{G}^{2}}} e^{j b_{G} t^{2}}
$$

and

$$
\int\left|x\left(t ; \boldsymbol{\theta}_{k}\right)\right|^{2} d t=1
$$

With this signal model, and considering $T<<$ PRI, the transmitted peak power is

$$
P_{p k}=\frac{E_{s}}{N_{P} \sqrt{\pi \lambda_{G}^{2}}}
$$

and the total transmitted energy is $E_{s}$. The received energy depends on the waveform parameter $\lambda_{G}$ and the range $r$ as

$$
E_{R}=\frac{P_{p k} \sqrt{\pi \lambda_{G}^{2}} G^{2} \sigma \lambda^{2}}{(4 \pi)^{3} r^{4}} N_{P}
$$

where $G$ is the antenna gain and $\sigma$ is the target Radar Cross Section (RCS). The power spectral density of the noise $N_{0}$ is only function of the receiver equivalent noise temperature $T_{e}$ as $N_{0}=k_{b} T_{e}$, where $k_{b}$ is the Boltzmann constant.

It can be shown that for a train of Gaussian chirps with $T<<$ PRI the FIM of time delay and Doppler can be approximated as [3] [29]

$$
\mathbf{J}^{(\tau, v)}=\operatorname{SNR}\left(\begin{array}{lr}
\frac{1}{2 \lambda_{G}^{2}}+2 \lambda_{G}^{2} b_{G}^{2} & 2 \pi \lambda_{G}^{2} b_{G} \\
2 \pi \lambda_{G}^{2} b_{G} & 2 \pi^{2} \lambda_{G}^{2}+\frac{1}{3} \pi^{2} \mathrm{PRI}^{2}\left(N_{p}^{2}-1\right)
\end{array}\right)
$$

We study the case when the sensing cost term $\Psi_{s}(k)$ is the transmitted energy at each step $E_{s}$. A number of strategies can be employed to account for the SNR for the assessment of performance.

1) The Signal to Noise ratio at each scan is obtained with the radar range equation (as in (26)) under the assumption that the transmitted peak power $P_{p k}$ remains constant at all times or that $P_{p k}$ can be automatically adapted to keep the energy of the transmitted signal constant. Under this hypotheses, $E_{R}$ is a function of the range $r$, which is a function of the state vector $\mathbf{e}_{k}$. As a result, the measurement noise is a function of the state and the solutions of the linear $\mathrm{KF}$, which requires 
independent measurement noise, are no longer valid. As a result, the proposed algorithm is not optimal. A solution of this type has been implemented in previous work (e.g. [3], [23] and [30]) and, as a result, the tracking performance achieved in these papers with the standard KF equations is also suboptimal.

2) The radar can automatically adapt certain parameters (e.g. the transmitted peak power) so to ensure that the received SNR at each scan remains constant. Under these assumptions, because SNR remains constant, the measurement noise covariance matrix does not depend on the state and the transmitted energy at each step, given a fixed range, does not depend on the waveform parameters. However, under this strategy, the transmitted energy

$$
E_{s}=\frac{(4 \pi)^{3} E_{R}}{G^{2} \sigma \lambda^{2}} r^{4}
$$

becomes a function of $r^{4}$ and therefore the assumption in Theorem 1 that $\Psi_{s}(k)$ is only a function of $\theta_{k}$ does not hold. Though, with this strategy, the algorithm presented in this paper remains optimal for $\alpha_{k}=0$.

The assumption of constant SNR is commonly employed to design modern radar systems that often have to perform multiple simultaneous tasks. Such a solution allows a better allocation and use of the available resources as well as the transmission of better Low Probability of Intercept (LPI) waveforms when the range decreases.

In a practical application, the radar will select the waveform parameters assuming constant SNR and will use the prediction of the range or the predicted mean value of the range to calculate the energy to transmit. Although, the measurement noise becomes a function of the estimate of the state, the robustness of the algorithm can be assessed with respect to resulting SNR fluctuations.

3) The radar can automatically adapt the transmitted peak power so to ensure that the received signal energy does not depend on range, i.e. that the ratio $P_{p k} / r^{4}$ remains constant. The energy of the received signal becomes

$$
E_{R}=\gamma \frac{\sqrt{\pi \lambda_{G}^{2}} G^{2} \sigma \lambda^{2}}{(4 \pi)^{3}} N_{P}
$$

with $\gamma$ being a constant, and the covariance matrix of the measurement noise does not depend on the state. Under this assumption, the transmitted energy depends on the waveform parameters and $r^{4}$ and, as for the previous strategy, the algorithm presented in this paper is optimal only for $\alpha_{k}=0$. The robustness of the algorithm with respect to fluctuations in SNR can be assessed by using the prediction or preticted mean value of the range as outlined for the strategy at constant SNR.

\section{Simulation Results}

A moving airborne platform is considered that carries a pulsed Doppler radar system operating at $10 \mathrm{GHz}$ with the task of reaching a target within 20 scans. The relative polar coordinates between the platform and the target are represented by

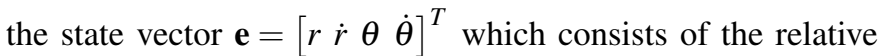
range $r$, relative radial velocity $\dot{r}$, relative angle $\theta$ and relative angle rate $\dot{\theta}$. The state equations are of the type used in [31] and [32] and described by the matrices

$$
\mathbf{F}=\left(\begin{array}{cccc}
1 & T & 0 & 0 \\
0 & 1 & 0 & 0 \\
0 & 0 & 1 & T \\
0 & 0 & 0 & 1
\end{array}\right) \quad \mathbf{Q}=\left(\begin{array}{ll}
\mathbf{Q}_{r} & \mathbf{0} \\
\mathbf{0} & \mathbf{Q}_{\theta}
\end{array}\right)
$$

with

$$
\mathbf{Q}_{r}=\sigma_{1}^{2}\left(\begin{array}{ll}
T^{3} / 3 & T^{2} / 2 \\
T^{2} / 2 & T
\end{array}\right) \quad \mathbf{Q}_{\theta}=\sigma_{2}^{2}\left(\begin{array}{ll}
T^{3} / 3 & T^{2} / 2 \\
T^{2} / 2 & T
\end{array}\right)
$$

$\sigma_{1}^{2}=0.01, \sigma_{2}^{2}=0.02$ and $T=1 \mathrm{~s}$. The control input is applied to all the elements of the state vector with

$$
\mathbf{B}=\mathbf{I}_{4 \times 4}
$$

and the radar measures the relative range, relative radial velocity and relative angle to the target with

$$
\mathbf{H}=\left(\begin{array}{llll}
1 & 0 & 0 & 0 \\
0 & 1 & 0 & 0 \\
0 & 0 & 1 & 0
\end{array}\right)
$$

It is assumed that the target has been positively detected and that all measurements are the result of true target associations. At each scan the radar transmits $N_{P}=2$ pulses with a PRI of $0.1 \mathrm{~ms}(\mathrm{PRF}=10 \mathrm{kHz})$. The transmitting and receiving antenna gain is $G=10 \mathrm{~dB}$ and the target RCS is $1 \mathrm{~m}^{2}$. The SNR is updated at each scan after calculating the received energy $E_{R}$ assuming a receiver temperature noise $T_{e}=1000 \mathrm{~K}^{\circ}$. The SNR strategy at constant $P_{p k} / r^{4}$ is employed to account for energy variations due the signal parameters and the constant $\gamma$ is obtained by setting a reference SNR of $0 \mathrm{~dB}$ for a pulsewidth of $1 \mu \mathrm{s}$. We also assume the transmitter and receiver employ separate antennas that point at the target with $\sigma_{\theta}^{2}=0.1 \mathrm{rad}^{2}$ so that the radar can transmit and receive at all times with no blind range. The initial covariance matrix is set to

$$
\mathbf{P}_{0 \mid 0}=\mathbf{I}_{4 \times 4}
$$

and the state vector at zero-time $\mathbf{e}_{0}$ is assumed known with elements equal to $1 \mathrm{~km},-1 \mathrm{~m} / \mathrm{s}, 0 \mathrm{rad}$ and $0 \mathrm{rad} / \mathrm{s}$. Finally, the weight $\alpha_{k}=2 \times 10^{6}$, the weight matrices are $\mathbf{E}=\mathbf{I}_{4 \times 4}$ and $\mathbf{R}=50 \times \mathbf{I}_{4 \times 4}$ and the matrix $\mathbf{S}_{N}$ is initialised with the solution of the ARE as in (13). The KF initial mean was set equal to zero. Note that, since $\alpha_{k} \neq 0$, the solution of the algorithm is suboptimal and because the SNR is time-variant the algorithm solution is non-stationary. Despite being suboptimal, the strategy offers anyway remarkable performance as shown in the simulation results.

Results relative to the adaptive case of a train of linear Gaussian chirps are compared with two other strategies:

$$
\begin{array}{ll}
\text { MinN: } & \min _{\boldsymbol{\theta}} \operatorname{Tr}(\mathbf{N}(\boldsymbol{\theta})) \\
\text { MinP: } & \min _{\boldsymbol{\theta}_{i}} \operatorname{Tr}\left(\mathbf{E} \mathbf{P}_{i \mid i}\right)
\end{array}
$$

In the first, the waveform parameters are selected so to minimize the trace of the measurement noise covariance matrix $\mathbf{N}(\boldsymbol{\theta})$, while, in the second, we minimise at each scan the 


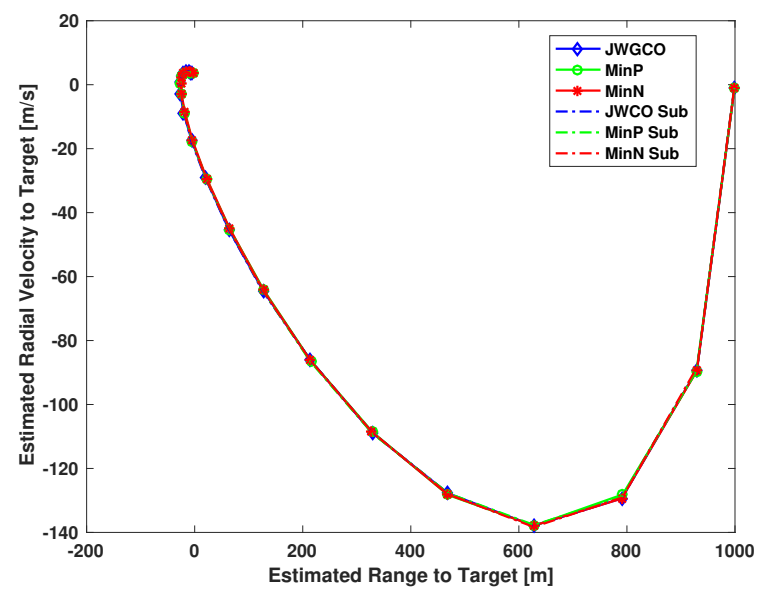

Fig. 2: Estimated relative velocity versus range to target for the three waveform selection strategies. The curves JWGCO Sub, MinP Sub and MinN Sub have been obtained using the prediction of the range.

trace of $\mathbf{P}_{i \mid i}$ weighted by the matrix $\mathbf{E}$. The bandwidth and pulsewidth of the chirp pulse at each scan are automatically selected by the algorithm in the range between $10 \mathrm{MHz}$ to 50 $\mathrm{MHz}$ and $1 \mu \mathrm{s}$ to $10 \mu \mathrm{s}$, respectively. In order to investigate the robustness of the algorithm with respect to SNR fluctuations, simulation results are also presented for the case when the radar uses the prediction of the range to calculate the peak power and therefore the energy to transmit. Figure 2 shows the estimated relative velocity versus the relative range to the target for the three waveform selection strategies. Results have been averaged over 500 Monte Carlo simulations. As expected, the interceptor reaches the target within the predefined number of scans for all cases. Figure 3 shows the optimal pulsewidth selected by the three strategies at each scan. Results show that the JWGCO algorithm selects the short pulsewidth at the beginning of the trajectory to switch to the longest pulsewidth in proximity of the target. The bandwidth remains fixed at all scans indicating no variation in range resolution (see Figure 4). Similar waveform selection results are obtained when the prediction of the range is used to calculate $P_{p k}$.

Figure 5 shows the corresponding ambiguity function of the waveforms transmitted along the trajectory and how it rotates from scan to scan for the JWGCO strategy. Results show the Doppler resolution is lower at the beginning of the trajectory, for the first six pulses, than that in proximity of the target. The range resolution remains the same throughout the trajectory as the transmitted bandwidth is constant. The range cut of the ambiguity function, showing a range resolution of $3 \mathrm{~m}$, is presented in Figure 6. Figure 7 and Figure 8 show the Doppler cuts of the AF for the first and last pulse of the transmitted sequence. Results show that, as expected, at the end of the sequence the Doppler resolution significantly improved and this is a direct consequence of employing a longer pulse. The key result is in Figure 9 which shows the averaged cumulative cost, i.e. the incremental value of (3) as $k \rightarrow N=20$ for the last 10 pulses. As expected, the strategy JWGCO achieves

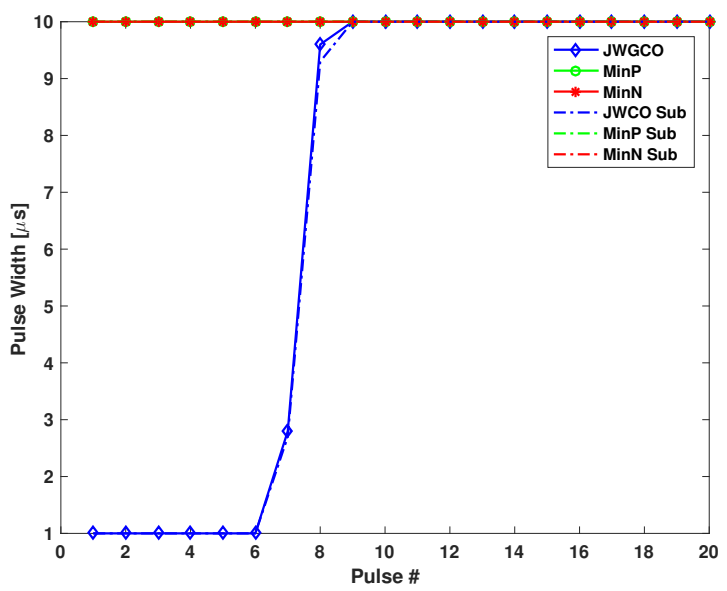

Fig. 3: Pulsewidth of the resulting linear chirp for the three waveform selection strategies at each scan.

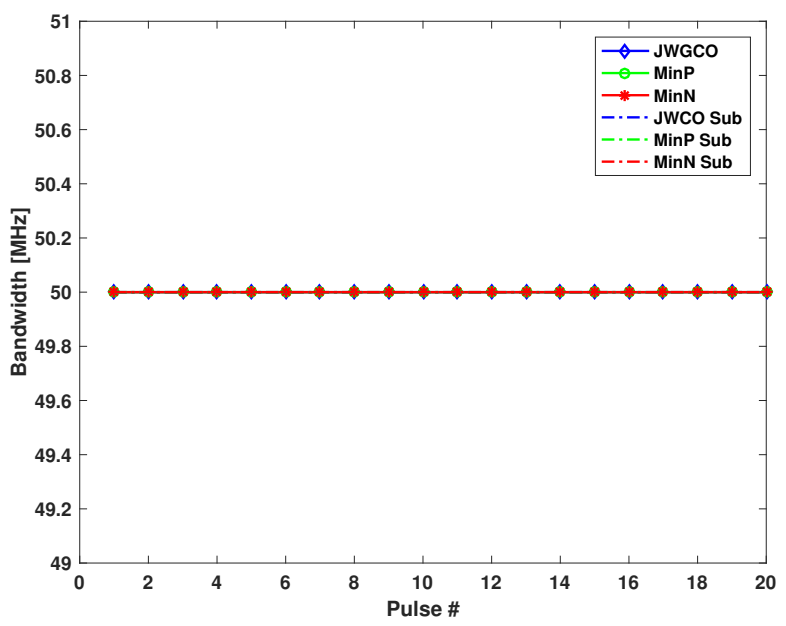

Fig. 4: Bandwidth of the resulting linear chirp for the three waveform selection strategies at each scan.

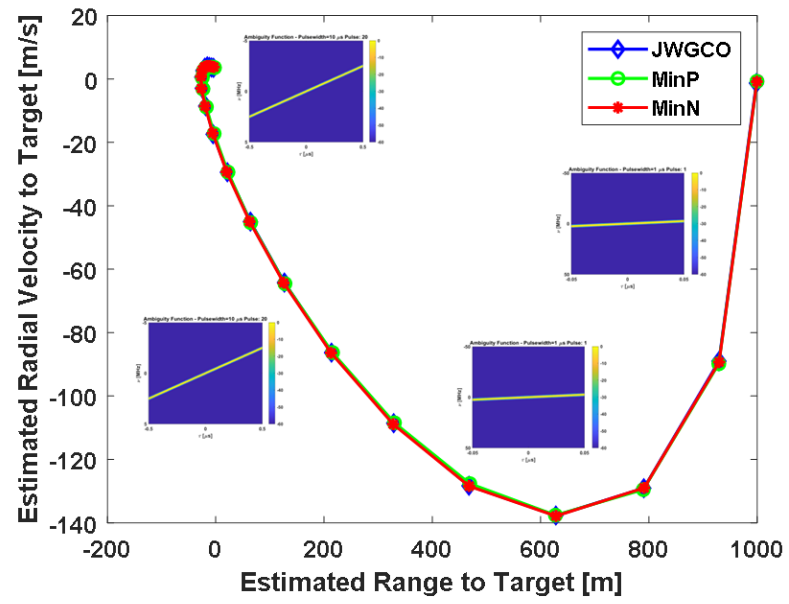

Fig. 5: Ambiguity Function of the resulting linear chirp for the JWGCO strategy at each scan. 


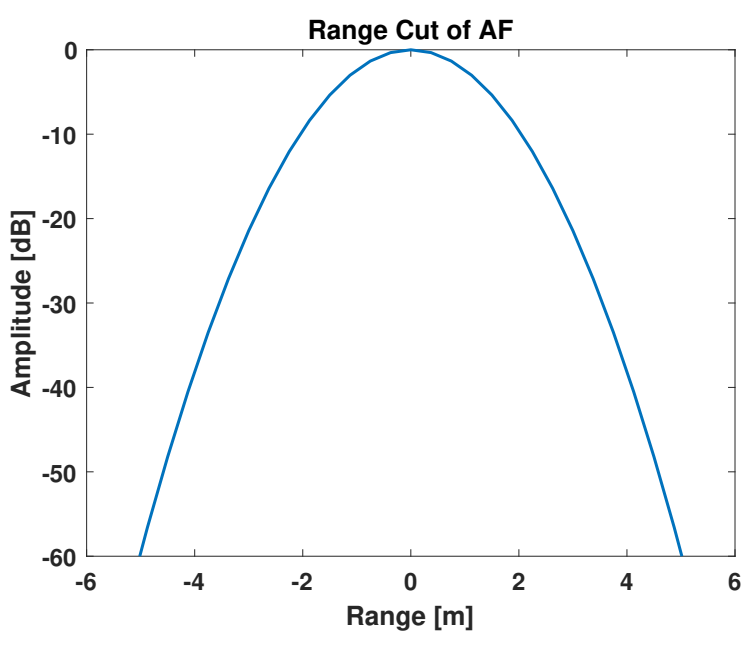

Fig. 6: Range Cut of the Ambiguity Function.

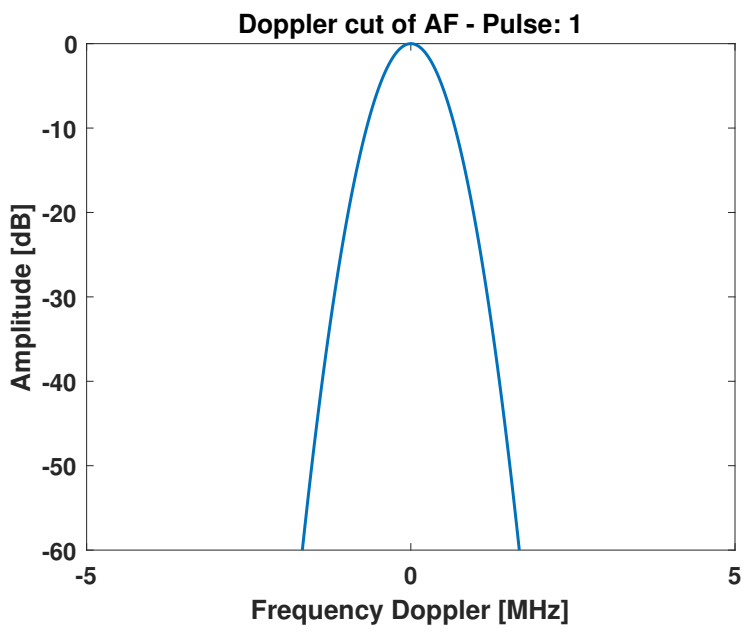

Fig. 7: Doppler Cut of the Ambiguity Function for the $1^{\text {st }}$ pulse.

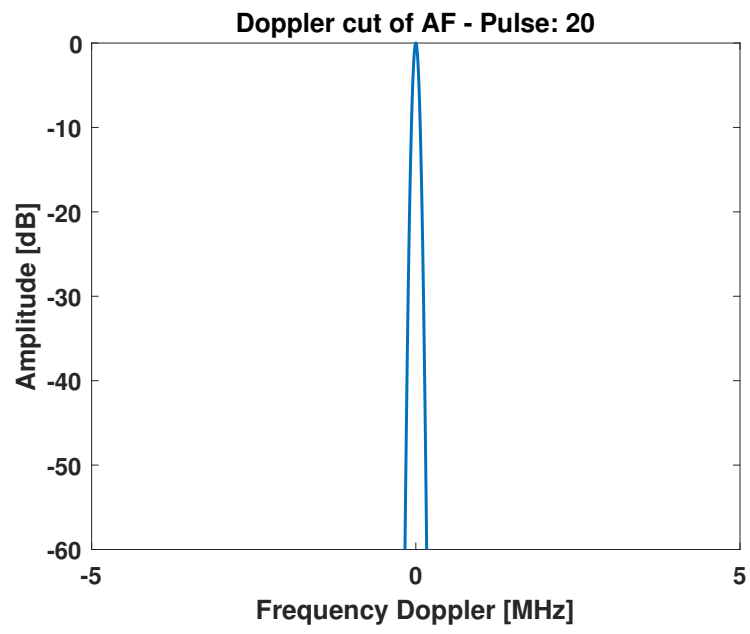

Fig. 8: Doppler Cut of the Ambiguity Function for the $20^{\text {th }}$ pulse.

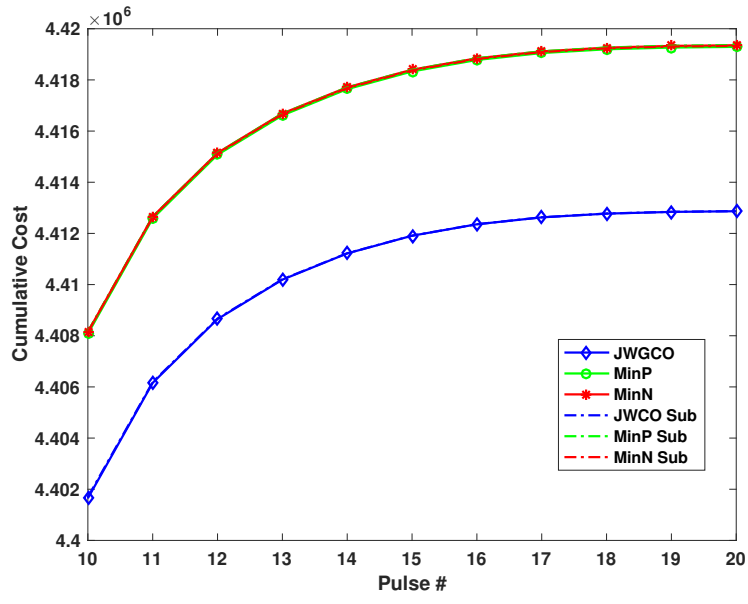

Fig. 9: Average cumulative cost for the three strategies.

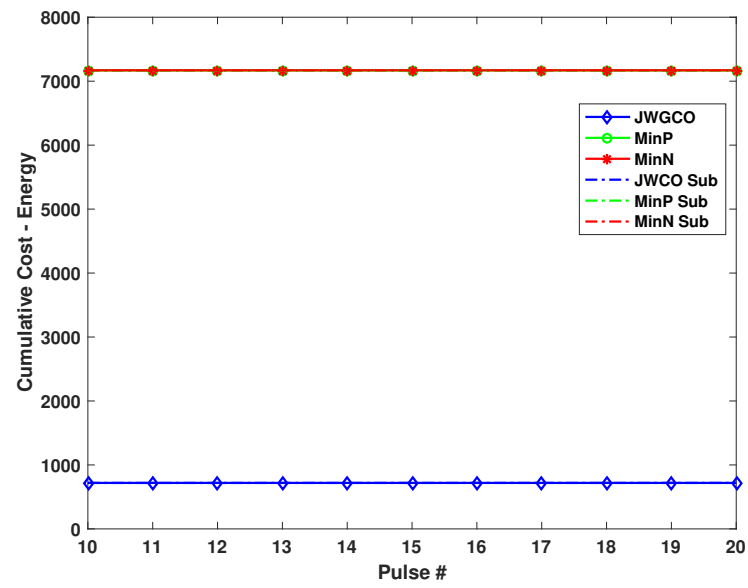

Fig. 10: Average cumulative cost of the term associated with the transmitted energy for the three strategies.

a lower final minimum cumulative cost with respect to the other two strategies. This was the main goal of the proposed algorithm. Results also show that performance is robust to SNR fluctuations. The contribution to the total cost of the transmitted energy, estimation errors and control input terms are shown in Figure 10, Figure 11 and Figure 12, respectively. Results show that the JWGCO results in a series of waveforms with the lowest transmitted energy.

Figure 13, Figure 14 and Figure 15 show the Root Mean square Error (RMSE) of the estimate of relative range, relative radial velocity and relative angle, respectively. Results show that the JWGCO algorithm allows for a larger RMSE of range and velocity at the beginning of the trajectory but then reaches similar values as those of the two other solutions in proximity of the target. The RMSE relative to the estimate of the angle is similar for all solutions.

\section{CONCLUSIONS}

The algorithm presented in this paper jointly selects the optimal transmitted waveform and the control input so that 


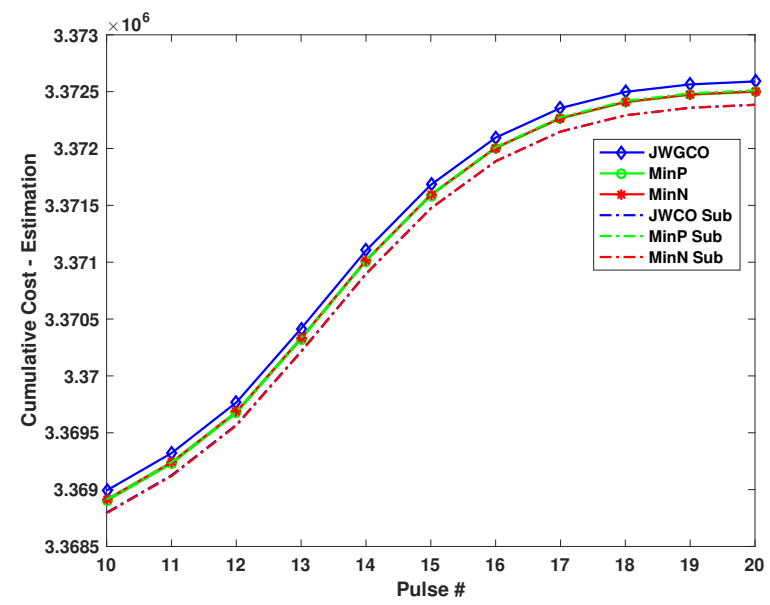

Fig. 11: Average cumulative cost of the term associated with the estimator covariance matrix for the three strategies.

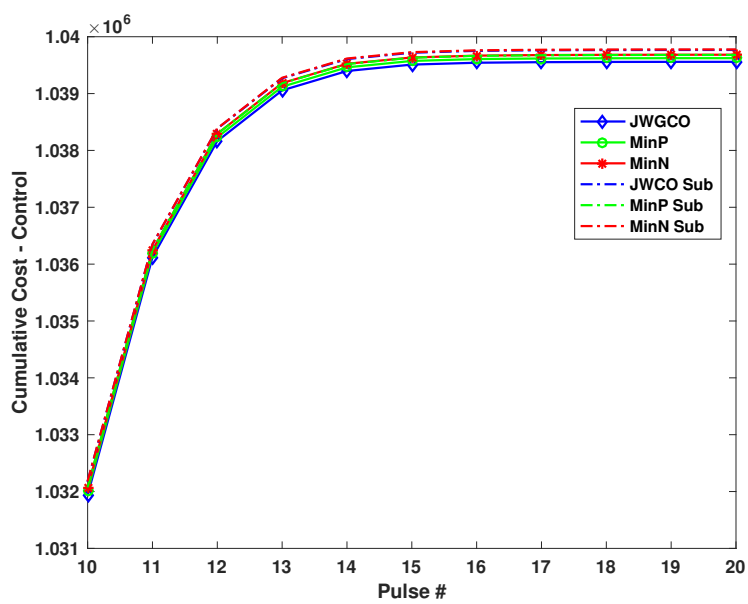

Fig. 12: Average cumulative cost of the term associated with the energy of the control input for the three strategies.

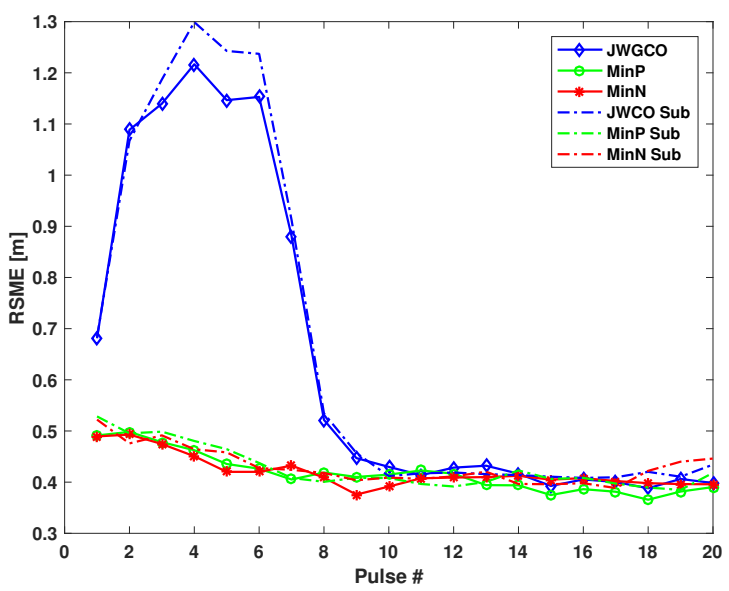

Fig. 13: Root Mean Square Error (RMSE) of relative range estimate.

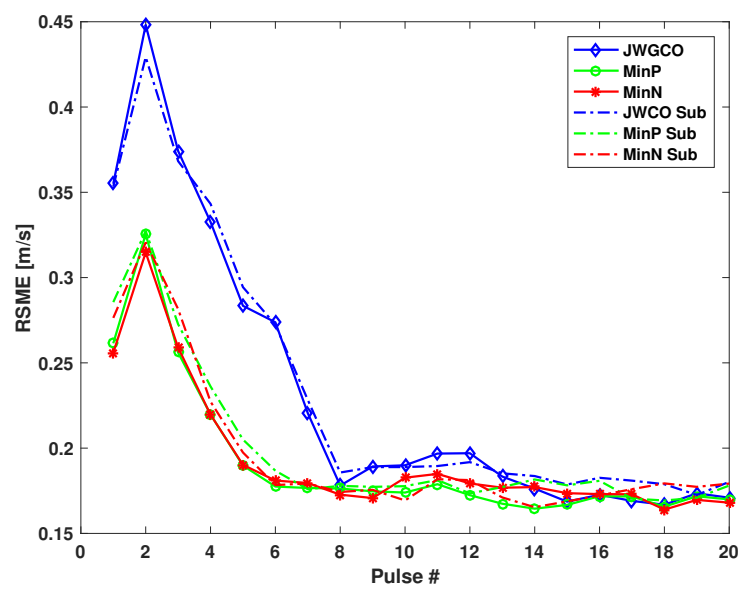

Fig. 14: Root Mean Square Error (RMSE) of relative velocity estimate.

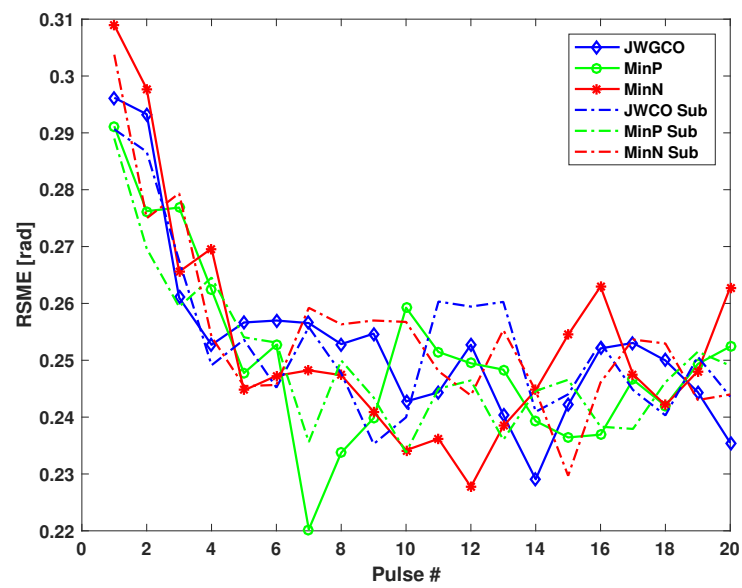

Fig. 15: Root Mean Square Error (RMSE) of relative angle estimate.

a sensor on a moving platform can intercept a target by minimising a cost function of the energy of the transmitted radar signal, the energy of the control input and the position error between the interceptor and the target. The solution achieves joint optimal waveform diversity and control and this is a contribution of this paper with respect to previous work where the two tasks were optimised separately. A set of simulations have been carried out to test the performance of the algorithm for a moving platform carrying a radar system operating at $10 \mathrm{GHz}$ and that transmits linear chirps with Gaussian envelopes.

Future work will investigate the extension of the algorithm to more realistic cases for both sonar and radar applications. The measurements of the target range and angle with respect to the sensor are non-linear functions of the 3D Cartesian coordinates of the target and this will be taken into account in future work together with more realistic chased trajectory escapes. In this case, since the optimal solution of waveform and guidance problem cannot be computed analytically, we 
plan to follow a different approach based on Model Predictive Control (receding horizon control). Although this approach is in general not optimal, in practice it can work very well [33] [24, Ch.9]. Extending the algorithm to account for nonstationary and compound clutter is a further key extension that, however, requires the development of a new theoretical framework to address the estimation performance of the target range and velocity in non-Gaussian clutter. The analysis of the role of angle is another extension that will require much more sophisticated state equations for the platform dynamics and (especially for non-linear state equations) the application of complex nonlinear dynamic programming. However, for linear state equations, Direction Of Arrival (DOA) performance can be taken into account with the JWGCO by introducing additional variables to the CRLB element representing the accuracy of the angle measurement. Eclipsing can be also taken into account to study the case of pulsed sensor systems [34]. The algorithm can also be extended to the wideband case to investigate if results similar to those shown in [19] for the case of an echolocating bat are obtainable. To do this, the study must be extended to a variety of waveform designs, such as hyperbolic chirps [29].

\section{APPENDIX A}

\section{PROOF OF THEOREM 1}

Let us consider the problem

$\min _{\mathbf{u}_{0: N-1}, \boldsymbol{\theta}_{1: N}} J\left(\mathbf{e}_{0}, \mathbf{u}_{0: N-1}, \boldsymbol{\theta}_{1: N}\right)=\min _{\mathbf{u}_{0: N-1}, \boldsymbol{\theta}_{1: N}} \mathscr{E}\left(\sum_{k=0}^{N} \ell\left(k, \mathbf{e}_{k}, \mathbf{u}_{k}, \boldsymbol{\theta}_{k}\right)\right)$

In the proof, we will repeatedly exploit the following general result

$$
\mathscr{E}\left(\mathbf{e}_{k}^{T} \mathbf{E}_{k} \mathbf{e}_{k} \mid \mathbf{z}_{1: k}, \mathbf{u}_{0: k-1}\right)=\hat{\mathbf{e}}_{k}^{T} \mathbf{E}_{k} \hat{\mathbf{e}}_{k}+\operatorname{Tr}\left(\mathbf{E}_{k} \mathbf{P}_{k \mid k}\right)
$$

where $\hat{\mathbf{e}}_{k}, \mathbf{P}_{k \mid k}$ are the $\mathrm{KF}$ mean and covariance matrix. We will also exploit the smoothing properties of conditional expectations

$$
\begin{aligned}
& \mathscr{E}\left(\mathscr{E}\left(\ell\left(k, \mathbf{e}_{k}, \mathbf{u}_{k}, \boldsymbol{\theta}_{k}\right) \mid \mathbf{z}_{1: k}, \mathbf{u}_{0: k-1}\right) \mid \mathbf{z}_{1: k-1}, \mathbf{u}_{0: k-2}\right) \\
& =\mathscr{E}\left(\ell\left(k, \mathbf{e}_{k}, \mathbf{u}_{k}, \boldsymbol{\theta}_{k}\right) \mid \mathbf{z}_{1: k-1}, \mathbf{u}_{0: k-2}\right)
\end{aligned}
$$

where $\mathbf{z}_{1: k}=\left\{\mathbf{z}_{1}, \ldots, \mathbf{z}_{k}\right\}$ and $\mathbf{u}_{0: k-1}=\left\{\mathbf{u}_{0}, \ldots, \mathbf{u}_{k-1}\right\}$.

Let us start from time $N$ with

$$
\begin{gathered}
\ell\left(N, \mathbf{e}_{N}, \mathbf{u}_{N}, \boldsymbol{\theta}_{N}\right)=\mathbf{e}_{N}^{T} \mathbf{E}_{N} \mathbf{e}_{N}+\operatorname{Tr}\left(\mathbf{E}_{N} \mathbf{P}_{N \mid N}\right) \\
=\mathbf{e}_{N}^{T} \mathbf{S}_{N} \mathbf{e}_{N}+\operatorname{Tr}\left(\mathbf{S}_{N} \mathbf{P}_{N \mid N}\right)+\alpha_{N} \Psi_{S}\left(\boldsymbol{\theta}_{N}\right)
\end{gathered}
$$

where we have introduced the matrix $\mathbf{S}_{N}=\mathbf{E}_{N}$. From the smoothing property, we have that

$$
\begin{aligned}
& \mathscr{E}\left(\ell\left(N-1, \mathbf{e}_{N-1}, \mathbf{u}_{N-1}, \boldsymbol{\theta}_{N-1}\right)\right. \\
& \left.+\mathscr{E}\left(\ell\left(N, \mathbf{e}_{N}, \mathbf{u}_{N}, \boldsymbol{\theta}_{N}\right) \mid \mathbf{z}_{1: N}, \mathbf{u}_{0: N-1}\right) \mid \mathbf{z}_{1: N-1}, \mathbf{u}_{0: N-2}\right) \\
& =\mathscr{E}\left(\ell\left(N-1, \mathbf{e}_{N-1}, \mathbf{u}_{N-1}, \boldsymbol{\theta}_{N-1}\right)\right. \\
& \left.+\ell\left(N, \mathbf{e}_{N}, \mathbf{u}_{N}, \boldsymbol{\theta}_{N}\right) \mid \mathbf{z}_{1: N-1}, \mathbf{u}_{0: N-2}\right)
\end{aligned}
$$

and by exploiting (1), we can write

$$
\begin{aligned}
& \ell\left(N, \mathbf{e}_{N}, \mathbf{u}_{N}, \boldsymbol{\theta}_{N}\right) \\
& =\left(\mathbf{F}_{N-1} \mathbf{e}_{N-1}+\mathbf{B}_{N-1} \mathbf{u}_{N-1}+\mathbf{w}_{N-1}\right)^{T} \mathbf{S}_{N}\left(\mathbf{F}_{N-1} \mathbf{e}_{N-1}\right. \\
& \left.+\mathbf{B}_{N-1} \mathbf{u}_{N-1}+\mathbf{w}_{N-1}\right)+\operatorname{Tr}\left(\mathbf{S}_{N} \mathbf{P}_{N \mid N}\right)+\alpha_{N} \Psi_{S}\left(\boldsymbol{\theta}_{N}\right)
\end{aligned}
$$

The calculations start by expressing the sum of the values of the cost function at time $N$ and $N-1$ as

$$
\begin{aligned}
& \ell\left(N-1, \mathbf{e}_{N-1}, \mathbf{u}_{N-1}, \boldsymbol{\theta}_{N-1}\right)+\ell\left(N, \mathbf{e}_{N}, \mathbf{u}_{N}, \boldsymbol{\theta}_{N}\right) \\
& =\mathbf{e}_{N-1}^{T} \mathbf{E}_{N-1} \mathbf{e}_{N-1}+\mathbf{u}_{N-1}^{T} \mathbf{R}_{N-1} \mathbf{u}_{N-1} \\
& +\left(\mathbf{F}_{N-1} \mathbf{e}_{N-1}+\mathbf{B}_{N-1} \mathbf{u}_{N-1}+\mathbf{w}_{N-1}\right)^{T} \mathbf{S}_{N} \\
& \left(\mathbf{F}_{N-1} \mathbf{e}_{N-1}+\mathbf{B}_{N-1} \mathbf{u}_{N-1}+\mathbf{w}_{N-1}\right)+\operatorname{Tr}\left(\mathbf{S}_{N} \mathbf{P}_{N \mid N}\right) \\
& +\alpha_{N} \Psi_{s}\left(\boldsymbol{\theta}_{N}\right)+\alpha_{N-1} \Psi_{S}\left(\boldsymbol{\theta}_{N-1}\right)
\end{aligned}
$$

followed by standard matrix algebraic operations and rearrangements to obtain

$$
\begin{aligned}
& =\mathbf{e}_{N-1}^{T}\left(\mathbf{E}_{N-1}+\mathbf{F}_{N-1}^{T} \mathbf{S}_{N} \mathbf{F}_{N-1}\right) \mathbf{e}_{N-1} \\
& +\mathbf{u}_{N-1}^{T}\left(\mathbf{R}_{N-1}+\mathbf{B}_{N-1}^{T} \mathbf{S}_{N} \mathbf{B}_{N-1}\right) \mathbf{u}_{N-1}+\alpha_{N-1} \Psi_{s}\left(\boldsymbol{\theta}_{N-1}\right) \\
& +\mathbf{e}_{N-1}^{T} \mathbf{F}_{N-1}^{T} \mathbf{S}_{N} \mathbf{B}_{N-1} \mathbf{u}_{N-1} \\
& +\mathbf{u}_{N-1}^{T} \mathbf{B}_{N-1}^{T} \mathbf{S}_{N} \mathbf{F}_{N-1} \mathbf{e}_{N-1}+\mathbf{w}_{N-1}^{T} \mathbf{S}_{N} \mathbf{w}_{N-1} \\
& +\left(\mathbf{u}_{N-1}^{T} \mathbf{B}_{N-1}^{T}+\mathbf{e}_{N-1}^{T} \mathbf{F}_{N-1}^{T}\right) \mathbf{S}_{N} \mathbf{w}_{N-1} \\
& +\mathbf{w}_{N-1}^{T} \mathbf{S}_{N}\left(\mathbf{B}_{N-1} \mathbf{u}_{N-1}+\mathbf{F}_{N-1} \mathbf{e}_{N-1}\right)+\operatorname{Tr}\left(\mathbf{S}_{N} \mathbf{P}_{N \mid N}\right)+\alpha_{N} \Psi_{s}\left(\boldsymbol{\theta}_{N}\right)
\end{aligned}
$$

The terms are $1 \times 1$ matrices are transposed to find the final expression becomes

$$
\begin{aligned}
& =\mathbf{e}_{N-1}^{T}\left(\mathbf{E}_{N-1}+\mathbf{F}_{N-1}^{T} \mathbf{S}_{N} \mathbf{F}_{N-1}\right) \mathbf{e}_{N-1} \\
& +\mathbf{u}_{N-1}^{T}\left(\mathbf{R}_{N-1}+\mathbf{B}_{N-1}^{T} \mathbf{S}_{N} \mathbf{B}_{N-1}\right) \mathbf{u}_{N-1}+\alpha_{N-1} \Psi_{s}\left(\boldsymbol{\theta}_{N-1}\right) \\
& +2 \mathbf{u}_{N-1}^{T} \mathbf{B}_{N-1}^{T} \mathbf{S}_{N} \mathbf{F}_{N-1} \mathbf{e}_{N-1}+\mathbf{w}_{N-1}^{T} \mathbf{S}_{N} \mathbf{w}_{N-1} \\
& +2 \mathbf{w}_{N-1}^{T} \mathbf{S}_{N}\left(\mathbf{F}_{N-1} \mathbf{e}_{N-1}+\mathbf{B}_{N-1} \mathbf{u}_{N-1}\right)+\operatorname{Tr}\left(\mathbf{S}_{N} \mathbf{P}_{N \mid N}\right)+\alpha_{N} \Psi_{s}\left(\boldsymbol{\theta}_{N}\right)
\end{aligned}
$$

The expectation of (43) is equal to

$$
\begin{aligned}
& \mathscr{E}\left(\mathbf{e}_{N-1}^{T}\left(\mathbf{E}_{N-1}+\mathbf{F}_{N-1}^{T} \mathbf{S}_{N} \mathbf{F}_{N-1}\right) \mathbf{e}_{N-1} \mid \mathbf{z}_{1: N-1}, \mathbf{u}_{0: N-2}\right) \\
& +\mathbf{u}_{N-1}^{T}\left(\mathbf{R}_{N-1}+\mathbf{B}_{N-1}^{T} \mathbf{S}_{N} \mathbf{B}_{N-1}\right) \mathbf{u}_{N-1} \\
& +\mathscr{E}\left(2 \mathbf{u}_{N-1}^{T} \mathbf{B}_{N-1}^{T} \mathbf{S}_{N} \mathbf{F}_{N-1} \mathbf{e}_{N-1} \mid \mathbf{z}_{1: N-1}, \mathbf{u}_{0: N-2}\right) \\
& +\mathscr{E}\left(\mathbf{w}_{N-1}^{T} \mathbf{S}_{N} \mathbf{w}_{N-1} \mid \mathbf{z}_{1: N-1}, \mathbf{u}_{0: N-2}\right) \\
& +\mathscr{E}\left(2 \mathbf{w}_{N-1}^{T} \mathbf{S}_{N}\left(\mathbf{F}_{N-1} \mathbf{e}_{N-1}+\mathbf{B}_{N-1} \mathbf{u}_{N-1}\right) \mid \mathbf{z}_{1: N-1}, \mathbf{u}_{0: N-2}\right) \\
& +\operatorname{Tr}\left(\mathbf{S}_{N} \mathbf{P}_{N \mid N}\right)+\alpha_{N-1} \Psi_{S}\left(\boldsymbol{\theta}_{N-1}\right)+\alpha_{N} \Psi_{S}\left(\boldsymbol{\theta}_{N}\right)
\end{aligned}
$$

By observing that

$$
\left\{\begin{array}{l}
\mathscr{E}\left(\mathbf{w}_{N-1} \mid \mathbf{z}_{1: N-1}, \mathbf{u}_{0: N-2}\right)=0 \\
\mathscr{E}\left(\mathbf{w}_{N-1}^{T} \mathbf{S}_{N} \mathbf{F}_{N-1} \mathbf{e}_{N-1} \mid \mathbf{z}_{1: N-1}, \mathbf{u}_{0: N-2}\right)=0 \\
\mathscr{E}\left(\mathbf{e}_{N-1} \mid \mathbf{z}_{1: N-1}, \mathbf{u}_{0: N-2}\right)=\hat{\mathbf{e}}_{N-1}^{T} \\
\mathscr{E}\left(\mathbf{w}_{k-1} \mathbf{w}_{k-1}^{T} \mid \mathbf{z}_{1: k-1}, \mathbf{u}_{0: k-2}\right)=\mathbf{Q}_{k}
\end{array}\right.
$$

we finally obtain

$$
\begin{aligned}
& =\hat{\mathbf{e}}_{N-1}^{T}\left(\mathbf{E}_{N-1}+\mathbf{F}_{N-1}^{T} \mathbf{S}_{N} \mathbf{F}_{N-1}\right) \hat{\mathbf{e}}_{N-1} \\
& +\operatorname{Tr}\left(\left(\mathbf{E}_{N-1}+\mathbf{F}_{N-1}^{T} \mathbf{S}_{N} \mathbf{F}_{N-1}\right) \mathbf{P}_{N-1 \mid N-1}\right) \\
& +\mathbf{u}_{N-1}^{T}\left(\mathbf{R}_{N-1}+\mathbf{B}_{N-1}^{T} \mathbf{S}_{N} \mathbf{B}_{N-1}\right) \mathbf{u}_{N-1} \\
& +2 \mathbf{u}_{N-1}^{T} \mathbf{B}_{N-1}^{T} \mathbf{S}_{N} \mathbf{F}_{N-1} \hat{\mathbf{e}}_{N-1}+\operatorname{Tr}\left(\mathbf{S}_{N} \mathbf{Q}_{N-1}\right) \\
& +\operatorname{Tr}\left(\mathbf{S}_{N} \mathbf{P}_{N \mid N}\right)+\alpha_{N-1} \Psi_{S}\left(\boldsymbol{\theta}_{N-1}\right)+\alpha_{N} \Psi_{S}\left(\boldsymbol{\theta}_{N}\right)
\end{aligned}
$$


Now observe that

$$
\begin{aligned}
& \min _{\mathbf{u}_{0: N-1}, \boldsymbol{\theta}_{1: N}} J\left(\mathbf{e}_{0}, \mathbf{u}_{0: N-1}, \boldsymbol{\theta}_{1: N}\right) \\
= & \min _{\mathbf{u}_{0: N-1}, \boldsymbol{\theta}_{1: N}} \mathscr{E}\left(\sum_{k=0}^{N-2} \ell\left(k, \mathbf{e}_{k}, \mathbf{u}_{k}, \boldsymbol{\theta}_{k}\right)\right. \\
+ & \mathscr{E}\left(\ell\left(N-1, \mathbf{e}_{N-1}, \mathbf{u}_{N-1}, \boldsymbol{\theta}_{N-1}\right)\right. \\
+ & \left.\left.\ell\left(N, \mathbf{e}_{N}, \mathbf{u}_{N}, \boldsymbol{\theta}_{N}\right) \mid \mathbf{z}_{1: N-1}, \mathbf{u}_{0: N-2}\right)\right) \\
= & \min _{\mathbf{u}_{0: N-2}, \boldsymbol{\theta}_{1: N-1}} \mathscr{E}\left(\sum_{k=0}^{N-2} \ell\left(k, \mathbf{e}_{k}, \mathbf{u}_{k}, \boldsymbol{\theta}_{k}\right)\right. \\
+ & \min _{\mathbf{u}_{N-1}, \boldsymbol{\theta}_{N}} \mathscr{E}\left(\ell\left(N-1, \mathbf{e}_{N-1}, \mathbf{u}_{N-1}, \boldsymbol{\theta}_{N-1}\right)\right. \\
+ & \left.\left.\ell\left(N, \mathbf{e}_{N}, \mathbf{u}_{N}, \boldsymbol{\theta}_{N}\right) \mid \mathbf{z}_{1: N-1}, \mathbf{u}_{0: N-2}\right)\right)
\end{aligned}
$$

By considering the inner minimisation, we can perform the minimisations w.r.t. $\mathbf{u}_{N-1}, \boldsymbol{\theta}_{N}$ independently, since the only quantity that depends on $\boldsymbol{\theta}_{N}$ is $\mathbf{P}_{N \mid N}$. The optimal control input $\mathbf{u}_{N-1}^{*}$ is obtained by taking the zero-point of the derivative w.r.t. $\mathbf{u}_{N-1}$ (see Appendix B) and is equal to

$$
\mathbf{u}_{N-1}^{*}=-\mathbf{L}_{N-1} \hat{\mathbf{e}}_{N-1}
$$

and the minimum w.r.t. $\boldsymbol{\theta}_{N}$ is

$$
\boldsymbol{\theta}_{N}^{*}=\arg \min _{\boldsymbol{\theta}_{N}} \operatorname{Tr}\left(\mathbf{S}_{N} \mathbf{P}_{N \mid N}\right)+\alpha_{N} \Psi_{S}\left(\boldsymbol{\theta}_{N}\right)
$$

We can then verify the other steps by induction. The calculation of the cost for the optimal parameters are given in Appendix C.

\section{APPENDIX B}

\section{CALCUlation OF THE OPTIMAL CONTROL INPUT}

The optimal control input $\mathbf{u}_{N-1}^{*}$ is obtained by calculating the zero-point of the derivative of (47) with respect to $\mathbf{u}_{N-1}$. After forcing to zero all the terms that are not a function of $\mathbf{u}_{N-1}$, this can be mathematically expressed as

$$
\begin{gathered}
\frac{\partial \mathbf{u}_{N-1}^{T}\left(\mathbf{R}_{N-1}+\mathbf{B}_{N-1}^{T} \mathbf{S}_{N} \mathbf{B}_{N-1}\right) \mathbf{u}_{N-1}}{\partial \mathbf{u}_{N-1}} \\
+2 \frac{\partial \mathbf{u}_{N-1}^{T} \mathbf{B}_{N-1}^{T} \mathbf{S}_{N} \mathbf{F}_{N-1} \hat{\mathbf{e}}_{N-1}}{\partial \mathbf{u}_{N-1}}=0
\end{gathered}
$$

The properties of matrix differentiation, for which if $\mathbf{x}$ and $\mathbf{y}$ are two vectors and $\mathbf{A}$ is a matrix then $\frac{\partial \mathbf{x}^{T} \mathbf{A x}}{\partial \mathbf{x}}=2 \mathbf{x}^{T} \mathbf{A}$ and $\frac{\partial \mathbf{x}^{T} \mathbf{A y}}{\partial \mathbf{x}}=\mathbf{y}^{T} \mathbf{A}^{T}$, are used to find the final expression

$$
\mathbf{u}_{N-1}=-\left(\mathbf{R}_{N-1}+\mathbf{B}_{N-1}^{T} \mathbf{S}_{N} \mathbf{B}_{N-1}\right)^{-1} \mathbf{B}_{N-1}^{T} \mathbf{S}_{N} \mathbf{F}_{N-1} \hat{\mathbf{e}}_{N-1}
$$

\section{APPENDIX C}

CALCULATION OF THE COST FUNCTION FOR THE OPTIMAL PARAMETERS

We replace $\mathbf{u}_{N-1}^{*}=-\mathbf{L}_{N-1} \hat{\mathbf{e}}_{N-1}$ and $\mathbf{P}_{N \mid N}^{*}=\mathbf{P}_{N \mid N}\left(\boldsymbol{\theta}_{N}^{*}\right)$ in (46) to obtain

$$
\begin{aligned}
& =\hat{\mathbf{e}}_{N-1}^{T}\left(\mathbf{E}_{N-1}+\mathbf{F}_{N-1}^{T} \mathbf{S}_{N} \mathbf{F}_{N-1}\right) \hat{\mathbf{e}}_{N-1} \\
& +\operatorname{Tr}\left(\left(\mathbf{E}_{N-1}+\mathbf{F}_{N-1}^{T} \mathbf{S}_{N} \mathbf{F}_{N-1}\right) \mathbf{P}_{N-1 \mid N-1}\right) \\
& +\hat{\mathbf{e}}_{N-1}^{T} \mathbf{L}_{N-1}^{T}\left(\mathbf{R}_{N-1}+\mathbf{B}_{N-1}^{T} \mathbf{S}_{N} \mathbf{B}_{N-1}\right) \mathbf{L}_{N-1} \hat{\mathbf{e}}_{N-1} \\
& -2 \hat{\mathbf{e}}_{N-1}^{T} \mathbf{L}_{N-1}^{T} \mathbf{B}_{N-1}^{T} \mathbf{S}_{N} \mathbf{F}_{N-1} \hat{\mathbf{e}}_{N-1} \\
& +\operatorname{Tr}\left(\mathbf{S}_{N} \mathbf{Q}_{N-1}\right)+\operatorname{Tr}\left(\mathbf{S}_{N} \mathbf{P}_{N \mid N}^{*}\right)+\alpha_{N-1} \Psi_{S}\left(\boldsymbol{\theta}_{N-1}\right)+\alpha_{N} \Psi_{S}\left(\boldsymbol{\theta}_{N}^{*}\right)
\end{aligned}
$$

which after some simple algebraic steps becomes

$$
\begin{aligned}
& =\hat{\mathbf{e}}_{N-1}^{T}\left(\mathbf{E}_{N-1}+\mathbf{F}_{N-1}^{T} \mathbf{S}_{N} \mathbf{F}_{N-1}-\mathbf{F}_{N-1}^{T} \mathbf{S}_{N} \mathbf{B}_{N-1}\right. \\
& \left.\left(\mathbf{B}_{N-1}^{T} \mathbf{S}_{N} \mathbf{B}_{N-1}+\mathbf{R}_{N-1}\right)^{-1} \mathbf{B}_{N-1}^{T} \mathbf{S}_{N} \mathbf{F}_{N-1}\right) \hat{\mathbf{e}}_{N-1} \\
& +\operatorname{Tr}\left(\left(\mathbf{E}_{N-1}+\mathbf{F}_{N-1}^{T} \mathbf{S}_{N} \mathbf{F}_{N-1}\right) \mathbf{P}_{N-1 \mid N-1}\right)+\operatorname{Tr}\left(\mathbf{S}_{N} \mathbf{P}_{N \mid N}^{*}\right) \\
& +\operatorname{Tr}\left(\mathbf{S}_{N} \mathbf{Q}_{N-1}\right)+\alpha_{N-1} \Psi_{S}\left(\boldsymbol{\theta}_{N-1}\right)+\alpha_{N} \Psi_{s}\left(\boldsymbol{\theta}_{N}^{*}\right)
\end{aligned}
$$

We define

$$
\begin{aligned}
\mathbf{S}_{N-1} & =\mathbf{E}_{N-1}+\mathbf{F}_{N-1}^{T} \mathbf{S}_{N} \mathbf{F}_{N-1}-\mathbf{F}_{N-1}^{T} \mathbf{S}_{N} \mathbf{B}_{N-1} \\
& \times\left(\mathbf{B}_{N-1}^{T} \mathbf{S}_{N} \mathbf{B}_{N-1}+\mathbf{R}_{N-1}\right)^{-1} \mathbf{B}_{N-1}^{T} \mathbf{S}_{N} \mathbf{F}_{N-1} \\
& =\mathbf{F}_{N-1}^{T}\left(\mathbf{S}_{N}-\mathbf{S}_{N} \mathbf{B}_{N-1}\left(\mathbf{B}_{N-1}^{T} \mathbf{S}_{N} \mathbf{B}_{N-1}+\mathbf{R}_{N-1}\right)^{-1} \mathbf{B}_{N-1}^{T} \mathbf{S}_{N}\right) \\
& \times \mathbf{F}_{N-1}+\mathbf{E}_{N-1}
\end{aligned}
$$

to express the cost as

$$
\begin{aligned}
& =\mathscr{E}\left(\mathbf{e}_{N-1}^{T} \mathbf{S}_{N-1} \mathbf{e}_{N-1} \mid \mathbf{u}_{0: N-1}, \mathbf{z}_{1: N-1}\right) \\
& +\operatorname{Tr}\left(\left(\mathbf{E}_{N-1}+\mathbf{F}_{N-1}^{T} \mathbf{S}_{N} \mathbf{F}_{N-1}-\mathbf{S}_{N-1}\right) \mathbf{P}_{N-1 \mid N-1}\right) \\
& +\operatorname{Tr}\left(\mathbf{S}_{N} \mathbf{P}_{N \mid N}^{*}\right)+\operatorname{Tr}\left(\mathbf{S}_{N} \mathbf{Q}_{N-1}\right)+\alpha_{N-1} \Psi_{S}\left(\boldsymbol{\theta}_{N-1}\right)+\alpha_{N} \Psi_{s}\left(\boldsymbol{\theta}_{N}^{*}\right)
\end{aligned}
$$

which equals

$$
\begin{aligned}
& =\mathscr{E}\left(\mathbf{e}_{N-1}^{T} \mathbf{S}_{N-1} \mathbf{e}_{N-1} \mid \mathbf{u}_{0: N-1}, \mathbf{z}_{1: N-1}\right) \\
& +\operatorname{Tr}\left(\mathbf{M}_{N-1} \mathbf{P}_{N-1 \mid N-1}\right) \\
& +\operatorname{Tr}\left(\mathbf{S}_{N} \mathbf{P}_{N \mid N}^{*}\right)+\operatorname{Tr}\left(\mathbf{S}_{N} \mathbf{Q}_{N-1}\right)+\alpha_{N-1} \Psi_{S}\left(\boldsymbol{\theta}_{N-1}\right)+\alpha_{N} \Psi_{S}\left(\boldsymbol{\theta}_{N}^{*}\right)
\end{aligned}
$$

with

$$
\mathbf{M}_{N-1}=\mathbf{E}_{N-1}+\mathbf{F}_{N-1}^{T} \mathbf{S}_{N} \mathbf{F}_{N-1}-\mathbf{S}_{N-1}
$$

where the equality before the last follows from the property in (36). We can then verify the other steps by induction.

\section{APPENDIX D}

\section{PROOF OF THEOREM 2}

The fact that $\mathbf{u}_{i}^{*}=-\mathbf{L} \hat{\mathbf{e}}_{i}$ where $\mathbf{L}$ is the constant-feedback matrix is a consequence of Proposition 1 and Theorem 1. For the second part, assume that such $\mathbf{P}^{*}$ exists, then if $\mathbf{P}_{0}=\tilde{\mathbf{P}}$ we have that the optimal $\boldsymbol{\theta}$ will satisfy $\boldsymbol{\theta}_{i}^{*}=\boldsymbol{\theta}^{*}$ for $i=1,2, \ldots$ and, therefore, $\mathbf{P}_{i}=\mathbf{P}^{*}$ for $i=1,2, \ldots$ 


\section{ACKNOWLEDGMENTS}

The authors thank Professor David Kershaw for providing his comments on the draft version of the paper and the anonymous Reviewers for the time they spent in reviewing the paper and for their valuable comments to improve the quality of the manuscript. Dr Balleri thanks the MCM-ITP programme for supporting his time on this work.

\section{REFERENCES}

[1] A. Farina, A. De Maio, and S. Haykin, Eds., The Impact of Cognition on Radar Technology, ser. Radar, Sonar and Navigation. Institution of Engineering and Technology, 2017.

[2] A. Charlish, K. Bell, N. Godman, and G. Smith, "Special issue on cognitive radar," IET Radar, Sonar and Navigation, vol. 12, no. 12, pp. 1361-1475, 2018

[3] D. Kershaw and R. Evans, "Optimal waveform selection for tracking systems," IEEE Transactions on Information Theory, vol. 40, no. 5, pp. 1536-1550, Sep 1994.

[4] N. H. Nguyen, K. Dogancay, and L. M. Davis, "Adaptive waveform selection for multistatic target tracking," IEEE Transactions on Aerospace and Electronic Systems, vol. 51, no. 1, pp. 688-701, January 2015.

[5] R. Niu, P. Willett, and Y. Bar-Shalom, "Tracking considerations in selection of radar waveform for range and range-rate measurements," IEEE Transactions on Aerospace and Electronic Systems, vol. 38, no. 2, pp. 467-487, Apr 2002.

[6] S. P. Sira, A. Papandreou-Suppappola, and D. Morrell, "Dynamic configuration of time-varying waveforms for agile sensing and tracking in clutter," IEEE Transactions on Signal Processing, vol. 55, no. 7, pp. 3207-3217, July 2007.

[7] M. Hurtado, T. Zhao, and A. Nehorai, "Adaptive polarized waveform design for target tracking based on sequential bayesian inference," IEEE Transactions on Signal Processing, vol. 56, no. 3, pp. 1120-1133, March 2008.

[8] K. L. Bell, C. J. Baker, G. E. Smith, J. T. Johnson, and M. Rangaswamy, "Cognitive radar framework for target detection and tracking," IEEE Journal of Selected Topics in Signal Processing, vol. 9, no. 8, pp. 1427 1439, Dec 2015.

[9] A. E. Mitchell, G. Smith, K. L. Bell, and M. Rangaswamy, "Cost function design for the fully adaptive radar framework," IET Radar, Sonar and Navigation, vol. 12, no. 12, pp. 1380-1389, 2018.

[10] S. Sen and A. Nehorai, "OFDM mimo radar with mutual-information waveform design for low-grazing angle tracking," IEEE Transactions on Signal Processing, vol. 58, no. 6, pp. 3152-3162, June 2010.

[11] R. Gui, W. Q. Wang, Y. Pan, and J. Xu, "Cognitive target tracking via angle-range-doppler estimation with transmit subaperturing FDA radar," IEEE Journal of Selected Topics in Signal Processing, vol. 12, no. 1, pp. 76-89, Feb 2018.

[12] J. Yan, B. Jiu, H. Liu, B. Chen, and Z. Bao, "Prior knowledge-based simultaneous multibeam power allocation algorithm for cognitive multiple targets tracking in clutter," IEEE Transactions on Signal Processing, vol. 63, no. 2, pp. 512-527, Jan 2015.

[13] S. Wang, D. Bi, H. Ruan, and S. Chen, "Cognitive structure adaptive particle filter for radar manoeuvring target tracking," IET Radar, Sonar and Navigation, vol. 13, no. 1, pp. 23-30, 2019.

[14] M. Hurtado and A. Nehorai, "Bat-inspired adaptive design of waveform and trajectory for radar," in 42nd Asilomar Conference on Signals, Systems and Computers, Oct 2008, pp. 36-40.

[15] M. Ben Kilani, Y. Nijsure, G. Gagnon, G. Kaddoum, and F. Gagnon, "Cognitive waveform and receiver selection mechanism for multistatic radar," IET Radar, Sonar Navigation, vol. 10, no. 2, pp. 417-425, 2016.

[16] C. Shi, F. Wang, M. Sellathurai, J. Zhou, and S. Salous, "Power minimization-based robust ofdm radar waveform design for radar and communication systems in coexistence," IEEE Transactions on Signal Processing, vol. 66, no. 5, pp. 1316-1330, March 2018.

[17] N. H. Nguyen, K. Doanay, and L. M. Davis, "Joint transmitter waveform and receiver path optimization for target tracking by multistatic radar system," in 2014 IEEE Workshop on Statistical Signal Processing (SSP), June 2014, pp. 444-447.

[18] C. Baker, H. Griffiths, and A. Balleri, "Biologically inspired waveform diversity (in Waveform Design and Diversity for Advanced Radar Systems)," Institution of Engineering and Technology, Series on Radar, Sonar, Navigation and Avionics, pp. 149-172, 2012.
[19] P. Flandrin, Animal Sonar: Processes and Performance. Boston, MA: Springer US, 1988, ch. Time-Frequency Processing of Bat Sonar Signals, pp. $797-802$.

[20] P. J. Nahin, Chases and escapes: the mathematics of pursuit and evasion. Princeton University Press, 2012.

[21] R. E. Kalman, "A new approach to linear filtering and prediction problems," Journal of Basic Engineering, vol. 82, no. 1, pp. 35-45, 1960.

[22] M. Athans, "On optimal allocation and guidance laws for linear interception and rendezvous problems," IEEE Transactions on Aerospace and Electronic Systems, vol. AES-7, no. 5, pp. 843-853, Sept 1971.

[23] A. Balleri, A. Farina, and A. Benavoli, "Coordination of optimal guidance law and adaptive radiated waveform for interception and rendezvous problems," IET Radar, Sonar and Navigation, vol. 11, no. 7, pp. 1132-1139, 2017.

[24] E. Mosca, Optimal, predictive, and adaptive control. Prentice-Hall, Inc., 1994.

[25] J. Lee and S. Leyffer, Mixed integer nonlinear programming. Springer Science \& Business Media, 2011, vol. 154.

[26] H. L. Van Trees, Detection, estimation and modulation theory, Part III. Radar-Sonar signal processing and Gaussian signals in noise. Wiley, 2001.

[27] C. E. Cook and M. Bernfeld, Radar Signals:an introduction to theory and application. Artech House, 1987.

[28] E. Kelly, "The radar measurement of range, velocity and acceleration," IRE Transactions on Military Electronics, vol. MIL-5, no. 2, pp. 51-57, April 1961.

[29] A. Balleri and A. Farina, "Ambiguity function and accuracy of the hyperbolic chirp: comparison with the linear chirp," IET Radar, Sonar and Navigation, vol. 11, no. 1, pp. 142-153, 2017.

[30] P. Stinco, M. S. Greco, F. Gini, and B. Himed, "ComRadE: Cognitive passive tracking in symbiotic IEEE 802.22 systems," IEEE Transactions on Aerospace and Electronic Systems, vol. 53, no. 2, pp. 1023-1034, April 2017.

[31] L. Ortenzi, L. Timmoneri, and D. Vigilante, "Unscented Kalman Filter (UKF) applied to FM band passive radar," in 2009 International Radar Conference "Surveillance for a Safer World" (RADAR 2009), Oct 2009, pp. 1-6.

[32] B. Ristic, S. Arulampalam, and N. Gordon, Beyond the Kalman Filter: Particle Filters for Tracking Applications. Artech House, 2004.

[33] A. Alessio and A. Bemporad, "A survey on explicit model predictive control," in Nonlinear model predictive control. Springer, 2009, pp. 345-369.

[34] M. I. Skolnik, Introduction to radar systems, 3rd ed. McGraw Hill, New York, 2011.

Alessio Benavoli received all his degrees in Computer and Control Engineering from the University of Firenze, Italy: the Ph.D in 2008 and the MS degree in 2004. He worked for one year (2008) for the international company SELEXSistemi Integrati as system analyst. From 2008 to 2019, he worked at the Dalle Molle Instititute for Artificial Intelligence (IDSIA) first as a researcher, then senior researcher, and finally professor. Currently, he is a Senior Lecturer at the Department of Computer Science and Information Systems (CSIS), University of Limerick, Ireland. His research interests are in the areas of filtering, machine learning, imprecise probabilities, Bayesian nonparametrics, decision-making under uncertainty and quantum theory. He has co-authored 80 peer-reviewed publications.

Alessio Balleri is a Senior Lecturer with Cranfield University at the Defence Academy of the United Kingdom, Shrivenham. From February 2010 until March 2012, he was a Research Associate in Radar Systems with the Department of Electronic and Electrical Engineering at University College London (UCL) and, from June 2004 to December 2004, he was a visiting Research Scholar with the Dept. of Electrical and Computer Engineering at University of Illinois at Chicago (UIC). He obtained a $\mathrm{PhD}$ degree in Electronic and Electrical Engineering from UCL in 2010 and a Laurea Degree (5 legal years, summa cum laude) from University of Pisa in 2004. 
Dr Balleri has guest co-edited a special issue on "Biologically Inspired Radar and Sonar Systems" for the IET Radar, Sonar and Navigation in 2012 and a special Issue on "Emerging Radar Techniques" for the EURASIP Journal on Advances in Signal Processing in 2013. He was the technical programme committee co-chair for the IET International Radar Conference 2017 (Belfast, UK) and is the technical co-chair of the 2020 IEEE International Radar Conference (Washington DC, USA). His research interests include radar and sonar system design, biologically inspired radar and sonar systems, adaptive radar, radar and sonar target classification, target feature extraction and modelling of radar clutter.

Alfonso Farina received the Doctoral degree in electronic engineering from the University of Rome, Rome, Italy, in 1973. In 1974, he joined Selenia, then Selex ES, where he became the Director of the Analysis of Integrated Systems Unit and, subsequently, the Director of Engineering of the Large Business Systems Division. In 2012, he was the Senior VP and the Chief Technology Officer (CTO) of the Company, reporting directly to the President. From 2013 to 2014, he was a Senior Advisor to the CTO. He retired in October 2014. From 1979 to 1985, he was also a Professor of radar techniques with the University of Naples, Italy. He is currently a Visiting Professor with the Department of Electronic and Electrical Engineering at University College London and with the Centre of Electronic Warfare, Information and Cyber at Cranfield University, a Distinguished Lecturer of the IEEE Aerospace and Electronic Systems Society and a Distinguished Industry Lecturer for the IEEE Signal Processing Society (Jan 2018-Dec 2019). He is a Consultant to Leonardo S.p.A. Land \& Naval Defence Electronics Division (Rome). He has authored or coauthored more than 700 peer-reviewed technical papers and books and monographs (published worldwide), some of them also translated in to Russian and Chinese. Dr. Farina has been an IEEE Signal Processing Magazine Senior Editorial Board Member (3-year term) since 2017. He was the Conference General Chairman of the IEEE RadarCon 2008, Rome, Italy, May 2008, and the Executive Conference Chair at the International Conference on Information Fusion, Florence, Italy, July 2006. He was the recipient of many awards, including Leader of the team that won the First Prize of the first edition of the Finmeccanica Award for Innovation Technology, out of more than 330 submitted projects by the Companies of Finmeccanica Group (2004); an International Fellow of the Royal Academy of Engineering, U.K. (2005), the fellowship was presented to him by HRH Prince Philip, the Duke of Edinburgh; IEEE Dennis J. Picard Medal for Radar Technologies and Applications for Continuous, Innovative, Theoretical, and Practical Contributions to Radar Systems and Adaptive Signal Processing Techniques (2010), IEEE Honor Ceremony, Montreal, Canada; Oscar Masi Award for the AULOS green radar by the Italian Industrial Research Association (AIRI) (2012); IET Achievement Medal for Outstanding contributions to radar system design, signal, data and image processing, and data fusion (2014); IEEE SPS Industrial Leader Award for contributions to radar array processing and industrial leadership (2017). In addition, he was the recipient of Best Paper Awards, such as B. Carlton of IEEE Transactions on Aerospace and Electronic Systems (2001, 2003, 2013), IET Proceeding on Radar Sonar and Navigation (2009 - 2010), and International Conference on Information Fusion (2004 and 2009). In the past few decades, he has been collaborating with several professional journals and conferences (mainly on radar and data fusion) as an Associate Editor, a Reviewer, an organizer of special issues, a Session Chairman, a Plenary Speaker, etc. He is a Fellow of the IEEE and IET, a Fellow of the Royal Academy of Engineering, and a Fellow of EURASIP.

The 26 October 2018 he was interviewed, among few top managers of the Company, at RAI Storia for the "70 anniversario di Leonardo Company" ("70th anniversary of Leonardo Company"). A 3 minute trailer is available at: https://www.youtube.com/watch?v=xYarcsoiyC8 